\title{
Constraining Nonperturbative Strong-Field Effects in Scalar-Tensor Gravity by Combining Pulsar Timing and Laser-Interferometer Gravitational-Wave Detectors
}

\author{
Lijing Shao, ${ }^{1, *}$ Noah Sennett, ${ }^{1,2}$ Alessandra Buonanno, ${ }^{1,2}$ Michael Kramer, ${ }^{3,4}$ and Norbert Wex ${ }^{3}$ \\ ${ }^{1}$ Max Planck Institute for Gravitational Physics (Albert Einstein Institute), \\ Am Mühlenberg 1, Potsdam D-14476, Germany \\ ${ }^{2}$ Department of Physics, University of Maryland, College Park, Maryland 20742, USA \\ ${ }^{3}$ Max-Planck-Institut für Radioastronomie, Auf dem Hügel 69, D-53121 Bonn, Germany \\ ${ }^{4}$ Jodrell Bank Centre for Astrophysics, The University of Manchester, Manchester M13 9PL, \\ United Kingdom
}

(Received 26 April 2017; revised manuscript received 17 July 2017; published 27 October 2017)

\begin{abstract}
Pulsar timing and laser-interferometer gravitational-wave $(\mathrm{GW})$ detectors are superb laboratories to study gravity theories in the strong-field regime. Here, we combine these tools to test the mono-scalartensor theory of Damour and Esposito-Farèse (DEF), which predicts nonperturbative scalarization phenomena for neutron stars (NSs). First, applying Markov-chain Monte Carlo techniques, we use the absence of dipolar radiation in the pulsar-timing observations of five binary systems composed of a NS and a white dwarf, and eleven equations of state (EOSs) for NSs, to derive the most stringent constraints on the two free parameters of the DEF scalar-tensor theory. Since the binary-pulsar bounds depend on the NS mass and the EOS, we find that current pulsar-timing observations leave scalarization windows, i.e., regions of parameter space where scalarization can still be prominent. Then, we investigate if these scalarization windows could be closed and if pulsar-timing constraints could be improved by laserinterferometer GW detectors, when spontaneous (or dynamical) scalarization sets in during the early (or late) stages of a binary NS (BNS) evolution. For the early inspiral of a BNS carrying constant scalar charge, we employ a Fisher-matrix analysis to show that Advanced LIGO can improve pulsar-timing constraints for some EOSs, and next-generation detectors, such as the Cosmic Explorer and Einstein Telescope, will be able to improve those bounds for all eleven EOSs. Using the late inspiral of a BNS, we estimate that for some of the EOSs under consideration, the onset of dynamical scalarization can happen early enough to improve the constraints on the DEF parameters obtained by combining the five binary pulsars. Thus, in the near future, the complementarity of pulsar timing and direct observations of GWs on the ground will be extremely valuable in probing gravity theories in the strong-field regime.
\end{abstract}

DOI: 10.1103/PhysRevX.7.041025

Subject Areas: Astrophysics, Gravitation, Particles and Fields

\section{INTRODUCTION}

In general relativity (GR), gravity is mediated solely by a rank-2 tensor, namely, the spacetime metric $g_{\mu \nu}$. Scalartensor theories of gravity, which add a scalar component to the gravitational interaction, are popular alternatives to GR. Though first proposed in 1921 [1], contemporary interest in these theories has been spurred by their potential connection to inflation and dark energy, as well as possible unified theories of quantum gravity [2]. A modern framework for the class of scalar-tensor theories we consider was

*lijing.shao@aei.mpg.de

Published by the American Physical Society under the terms of the Creative Commons Attribution 4.0 International license. Further distribution of this work must maintain attribution to the author(s) and the published article's title, journal citation, and DOI. developed in Refs. [3-8] (see also more generic Horndeski scalar-tensor theories in Ref. [9]).

Ultimately, the existence (or absence) of scalar degrees of freedom (d.o.f.) in gravity will be decided by experiments. Most scalar-tensor theories are designed to be metric theories of gravity; that is, they respect the Einstein equivalence principle $[8,10]$. Therefore, precision tests of the weak-equivalence principle, the local Lorentz invariance, and the local position invariance in flat spacetime are unable to constrain them [8,10-12]. However, such theories generally violate the strong-equivalence principle. Tests of the strong-equivalence principle with self-gravitating bodies provide an ideal window to experimentally search for (or rule out) the scalar sector of gravity $[8,10,13]$.

Particularly prominent violations of the strongequivalence principle are known to arise in the class of massless mono-scalar-tensor theories, studied by Damour and Esposito-Farèse in the form of nonperturbative strongfield effects in neutron stars (NSs) [14-16]. In this paper, 
we investigate the extent to which pulsar timing and ground-based gravitational-wave (GW) observations can constrain these phenomena (space-based GW experiments [17-19] are beyond the scope of this paper). Our results demonstrate that, depending on the parameters of binary systems and NS equations of state (EOSs), these two types of experiments can provide complementary bounds on scalar-tensor theories [10-12,20,21]. These results are especially timely as new instruments come online in the upcoming years in both fields [22,23].

The paper is organized as follows. In the next section, we briefly review two nonperturbative phenomena (notably, spontaneous scalarization $[14,15]$ and dynamical scalarization [20,24-27]) that arise in certain scalar-tensor theories of gravity. Then, in Sec. III, we derive stringent constraints on these theories by combining state-of-the-art pulsar observations of five NS-white dwarf (WD) systems. In Sec. IV, we employ these constraints and investigate the potential detectability of nonperturbative effects in binary NS (BNS) systems using the Advanced Laser Interferometer Gravitational-Wave Observatory (LIGO) [28] and next-generation ground-based detectors. Finally, in Sec. V, we discuss the main results and implications of our findings and give perspectives for future observations.

\section{NONPERTURBATIVE STRONG-FIELD PHENOMENA IN SCALAR-TENSOR GRAVITY}

In this paper, we focus on the class of mono-scalar-tensor theories that are defined by the following action in the Einstein frame $[4,5,14,15]$,

$$
\begin{aligned}
S= & \frac{c^{4}}{16 \pi G_{*}} \int \frac{\mathrm{d}^{4} x}{c} \sqrt{-g_{*}}\left[R_{*}-2 g_{*}^{\mu \nu} \partial_{\mu} \varphi \partial_{\nu} \varphi-V(\varphi)\right] \\
& +S_{m}\left[\psi_{m} ; A^{2}(\varphi) g_{\mu \nu}^{*}\right],
\end{aligned}
$$

where $G_{*}$ is the bare gravitational coupling constant, $g_{\mu \nu}^{*}$ is the Einstein metric with its determinant $g_{*}, R_{*} \equiv g_{*}^{\mu \nu} R_{\mu \nu}^{*}$ is the Ricci scalar, $\psi_{m}$ collectively denotes the matter content, and $A(\varphi)$ is the (conformal) coupling function that depends on the scalar field $\varphi$. Henceforth, for simplicity, we assume that the potential $V(\varphi)$ is a slowly varying function that changes on scales much larger than typical length scales of the system that we consider; thus, we set $V(\varphi)=0$ in our calculation.

The field equations are derived with the least-action principle [7,8] for $g_{\mu \nu}^{*}$ and $\varphi$,

$$
\begin{gathered}
R_{\mu \nu}^{*}=2 \partial_{\mu} \varphi \partial_{\nu} \varphi+\frac{8 \pi G_{*}}{c^{4}}\left(T_{\mu \nu}^{*}-\frac{1}{2} T^{*} g_{\mu \nu}^{*}\right), \\
\square_{g^{*}} \varphi=-\frac{4 \pi G_{*}}{c^{4}} \alpha(\varphi) T_{*},
\end{gathered}
$$

with the energy-momentum tensor of matter fields, $T_{*}^{\mu \nu} \equiv 2 c\left(-g_{*}\right)^{-1 / 2} \delta S_{m} / \delta g_{\mu \nu}^{*}$, and the field-dependent coupling strength between the scalar field and the trace of the energy-momentum tensor of matter fields, $\alpha(\varphi) \equiv \partial \ln A(\varphi) / \partial \varphi$.

Following Damour and Esposito-Farèse [7,15], we consider a polynomial form for $\ln A(\varphi)$ up to quadratic order, that is, $A(\varphi)=\exp \left(\beta_{0} \varphi^{2} / 2\right)$, and denote $\alpha_{0} \equiv \alpha\left(\varphi_{0}\right)=$ $\beta_{0} \varphi_{0}$, with $\varphi_{0}$ the asymptotic value of $\varphi$ at infinity. This particular scalar-tensor theory (henceforth, DEF theory) is completely characterized by two parameters $\left(\alpha_{0}, \beta_{0}\right)$, and for systems dominated by strong-field gravity, such as NSs, it can give rise to potentially observable, nonperturbative physical phenomena $[14,24]$. Weak-field Solar System experiments, generally, only probe the $\alpha_{0}$ dimension or the combination $\beta_{0} \alpha_{0}^{2}$ in the $\left(\alpha_{0}, \beta_{0}\right)$ parameter space (see Refs. $[10,29]$ and references therein). In GR, $\alpha_{0}=\beta_{0}=0$.

Using a perfect-fluid description of the energy-momentum tensor for NSs in the Jordan frame, in 1993 Damour and Esposito-Farèse derived the Tolman-Oppenheimer-Volkoff (TOV) equations [14] for a NS in their scalar-tensor gravity theory. Interestingly, they discovered a phase-transition phenomenon when $\beta_{0} \lesssim-4$, largely irrespective of the $\alpha_{0}$ value (a nonzero $\alpha_{0}$ tends to smooth the phase transition [15]). The phenomenon was named spontaneous scalarization. With a suitable $\left(\alpha_{0}, \beta_{0}\right)$, the "effective scalar coupling" that a NS develops, $\alpha_{A} \equiv \partial \ln m_{A} / \partial \varphi_{0}$ (the baryonic mass of NS is fixed while taking the derivative), could be $\mathcal{O}(1)$ when the NS mass $m_{A}$ is within a certain EOS-dependent range [30]. For masses below and above this range, the effective scalar coupling is much smaller [32]. In Fig. 1, we show an example of spontaneous scalarization for a NS with the realistic EOS SLy4 and compare it to existing individual binary-pulsar constraints.

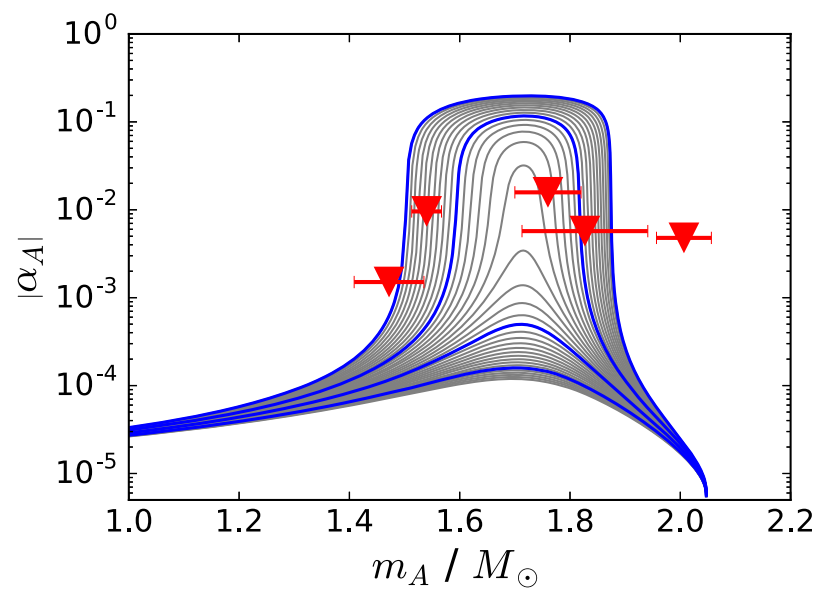

FIG. 1. Illustration of spontaneous scalarization in the DEF gravity, in comparison to individual binary-pulsar limits, for a NS with EOS SLy4 and $\left|\alpha_{0}\right|=10^{-5}$. The blue curves correspond to (from top to bottom) $\beta_{0}=-4.5,-4.4,-4.3$, and -4.2 ; the grey curves in between differ in $\beta_{0}$ in steps of 0.01 . We indicate with triangles the $90 \%$ C.L. upper limits on the effective scalar coupling $\left|\alpha_{A}\right|$ from the individual pulsars listed in Table I. We can clearly see a scalarization window at $m_{A} \sim 1.7 M_{\odot}$. 
In general, if two compact bodies in a binary have effective scalar couplings, $\alpha_{A}$ and $\alpha_{B}$, they produce gravitational dipolar radiation $\propto(\Delta \alpha)^{2}$, with $\Delta \alpha \equiv \alpha_{A}-\alpha_{B}$, which is at a lower post-Newtonian (PN) order than the canonical quadrupolar radiation in GR [15,33]. In Ref. [16], Damour and Esposito-Farèse, for the first time, compared limits on the DEF gravity arising from Solar System and binary pulsar experiments with expected limits from ground-based GW detectors like LIGO and Virgo. The analysis in Ref. [16] is based on soft (by now excluded [34,35]), medium, and stiff EOSs, and for the LIGO/Virgo experiment, it assumes a BNS merger with PSR B1913 + 16-like masses $\left(1.44 M_{\odot}\right.$ and $\left.1.39 M_{\odot}\right)$, as well as a $1.4 M_{\odot}-10 M_{\odot}$ NS-BH merger. Damour and Esposito-Farèse come to the conclusion that binary-pulsar experiments would generally be expected to put more stringent constraints on the parameters $\left(\alpha_{0}, \beta_{0}\right)$ than ground-based detectors, such as LIGO and Virgo. Since then, several analyses have followed [35-39], but typically, those studies did not probe a large set of NS masses and EOSs. Considering advances in our knowledge of NSs and more sensitive current and future ground-based detectors, we revisit this study here. Quite interestingly, as pointed out in a first study in Ref. [20], the constraints on the parameters $\left(\alpha_{0}, \beta_{0}\right)$ from binary pulsars depend quite crucially on the EOSs and the masses of the NSs, in particular, in the parameter space that allows for spontaneous scalarization. By taking into account this dependence when setting bounds from pulsar timing, we find that current and future GW detectors on the Earth might still be able to exclude certain specific regions of the parameter space $\left(\alpha_{0}, \beta_{0}\right)$ that have not been probed by binary pulsars yet.

Twenty years after the discovery of spontaneous scalarization, Barausse et al. [24] found another interesting nonperturbative phenomenon in a certain parameter space of the DEF theory. In this case, the scalarization does not take place for a NS in isolation but for NSs in a merging binary. Indeed, modeling the BNS evolution in numerical relativity, Barausse et al. found that the two NSs can scalarize even if initially, at large separation, they are not scalarized. This phenomenon is called dynamical scalarization, and its onset is determined by the binary compactness instead of the NS compactness. Reference [24] also demonstrated that a spontaneously scalarized NS can generate scalar hair on its initially unscalarized NS companion in a binary system through a process known as induced scalarization. Dynamical and induced scalarization cause BNSs to merge earlier $[20,24,26]$ than in GR, resulting in a significant modification to the GW phasing that is potentially detectable by ground-based GW interferometers $[24,25,27,40]$.

Finally, it is important to note that cosmological solutions in the strictly massless limit of the DEF theory are known to evolve away from GR when $\beta_{0}$ is negative
[40-43]; to be consistent with current Solar System observations, such cosmologies require significant finetuning of initial conditions [44]. Various modifications to the theory have been considered to cure this fine-tuning problem, e.g., adding higher-order polynomial terms to $\ln A(\varphi)$ [43] or including a mass term $V(\varphi)=2 m_{\varphi} \varphi^{2}$ in the action [15,46-48]. To date, none of these proposals has produced scalar-tensor theories that (i) satisfy cosmological and weak-field gravity constraints, (ii) generate an asymptotic field $\varphi_{0}$ that is stable over time scales relevant to binary pulsars and GW sources, and (iii) give rise to the nonperturbative phenomena present in DEF theory. As is commonly done in the literature $[7,14,15,20,24-27,40]$, we ignore these cosmological concerns in this paper and focus only on (massless) DEF theory.

\section{CONSTRAINTS FROM BINARY PULSARS}

Until now, binary pulsars have provided the most stringent limits to the DEF theory [15,35,38,39,49]. These limits were usually obtained with individual pulsar systems and with representative EOSs [39,50]. Here, by contrast, we combine observational results from multiple pulsar systems employing Markov-chain Monte Carlo (MCMC) simulations [52]. In particular, we pick the five NS-WD binaries that are the most constraining systems in testing spontaneous scalarization: PSRs J0348+0432 [35], J1012 + 5307 [53], J1738 + 0333 [38], J1909 3744 [54], and J2222 - 0137 [49]. We choose these five binaries based on the binary nature (namely, NS-WD binaries), the timing precision that has been achieved, and the NS masses. These aspects are important to the study here; see Refs. [11,12,51] for more discussions. For convenience, we list the parameters of these binaries in Table I, and we notice that it is the combination of their $\dot{P}_{b}^{\text {int }}$ and the NS mass that makes them particularly suitable for the test of spontaneous scalarization. We obtain the limits using 11 different EOSs that have a maximum NS mass above $2 M_{\odot}$ [55]. The names of these EOSs are AP3, AP4, ENG, H4, MPA1, MS0, MS2, PAL1, SLy4, WFF1, and WFF2 (see Refs. [55,56] for reviews). Figure 2 shows the massradius relation of NSs in GR for these EOSs. As evidenced by the spread of the curves in the figure, we believe that these EOSs are sufficient to cover the different EOSdependent properties of spontaneous scalarization and, at the same time, satisfy the two-solar-mass limit from pulsartiming observations $[34,35]$.

Markov-chain Monte Carlo techniques allow us to preform parameter estimation within the Bayesian framework. These methods provide the posterior distributions of the underlying parameters that are consistent with observations. In Bayesian analysis, given data $\mathcal{D}$ and a hypothesis $\mathcal{H}$ (here, the DEF theory), the marginalized posterior distribution of $\left(\alpha_{0}, \beta_{0}\right)$ is given by [52] 
TABLE I. Binary parameters of the five NS-WD systems that we use to constrain the DEF theory $[35,38,49,53,54]$. The observed time derivatives of the orbit period $P_{b}$ are corrected using the latest Galactic potential of Ref. [57]. For PSRs J0348 +0432 , J1012 + 5307, and $\mathrm{J} 1738+0333$, the mass ratios were obtained by combining radio timing and optical high-resolution spectroscopy, while the companion masses are determined from the Balmer lines of the WD spectra based on WD models [35,58-60]. For PSRs J1909 - 3744 and J2222 - 0137, the masses were calculated from the Shapiro delay, where the range of the Shapiro delay gives the companion mass directly, and the pulsar mass is then derived from the mass function, using the shape of the observed Shapiro delay to determine the orbital inclination [49,54]. The masses below are based on GR as the underlying gravity theory. However, since the companion WD is a weakly self-gravitating body, they are practically the same in the DEF theory (with a difference $\lesssim 10^{-4}$ ). In parentheses, we give the standard $1 \sigma$ errors in units of the least significant digit(s).

\begin{tabular}{lccccc}
\hline \hline Pulsar & $\mathrm{J} 0348+0432[35]$ & $\mathrm{J} 1012+5307[53]$ & $\mathrm{J} 1738+0333[38]$ & $\mathrm{J} 1909-3744[54]$ & $\mathrm{J} 2222-0137[49]$ \\
\hline Orbital period, $P_{b}(\mathrm{~d})$ & $0.102424062722(7)$ & $0.60467271355(3)$ & $0.3547907398724(13)$ & $1.533449474406(13)$ & $2.44576454(18)$ \\
Eccentricity, $e$ & $2.6(9) \times 10^{-6}$ & $1.2(3) \times 10^{-6}$ & $3.4(11) \times 10^{-7}$ & $1.14(10) \times 10^{-7}$ & $0.00038096(4)$ \\
Observed $\dot{P}_{b}, \dot{P}_{b}^{\text {obs }}\left(\mathrm{fs} \mathrm{s}^{-1}\right)$ & $-273(45)$ & $-50(14)$ & $-17.0(31)$ & $-503(6)$ & $200(90)$ \\
Intrinsic $\dot{P}_{b}, \dot{P}_{b}^{\text {int }}\left(\mathrm{fs} \mathrm{s}^{-1}\right)$ & $-274(45)$ & $-5(9)$ & $-27.72(64)$ & $-6(15)$ & $\ldots$ \\
Mass ratio, $q \equiv m_{p} / m_{c}$ & $11.70(13)$ & $10.5(5)$ & $8.1(2)$ & $\ldots$ & $1.540(27)$ \\
Pulsar mass, $m_{p}^{\text {obs }}\left(M_{\odot}\right)$ & $\ldots$ & $\ldots$ & $0.170(24)$ & $1.76(6)$ \\
WD mass, $m_{c}^{\text {obs }}\left(M_{\odot}\right)$ & $0.1715_{-0.0030}^{+0.0045}$ & $0.174(7)$ & $0.1817_{-0.0054}^{+0.0073}$ & $0.2130(24)$ & $1.293(25)$ \\
\hline \hline
\end{tabular}

$$
\begin{aligned}
& P\left(\alpha_{0}, \beta_{0} \mid \mathcal{D}, \mathcal{H}, \mathcal{I}\right) \\
& \quad=\int \frac{P\left(\mathcal{D} \mid \alpha_{0}, \beta_{0}, \boldsymbol{\Xi}, \mathcal{H}, \mathcal{I}\right) P\left(\alpha_{0}, \beta_{0}, \boldsymbol{\Xi} \mid \mathcal{H}, \mathcal{I}\right)}{P(\mathcal{D} \mid \mathcal{H}, \mathcal{I})} \mathrm{d} \boldsymbol{\Xi},
\end{aligned}
$$

where $\mathcal{I}$ is all other relevant prior background knowledge and $\boldsymbol{\Xi}$ collectively denotes all other unknown parameters besides $\left(\alpha_{0}, \beta_{0}\right)$, which are marginalized over to obtain the marginalized posterior distributions for just $\left(\alpha_{0}, \beta_{0}\right)$ (see below for more details). In the above equation, given $\mathcal{H}$ and $\mathcal{I}, P\left(\alpha_{0}, \beta_{0}, \boldsymbol{\Xi} \mid \mathcal{H}, \mathcal{I}\right)$ is the prior on $\left(\alpha_{0}, \beta_{0}, \boldsymbol{\Xi}\right)$, $P\left(\mathcal{D} \mid \alpha_{0}, \beta_{0}, \boldsymbol{\Xi}, \mathcal{H}, \mathcal{I}\right) \equiv \mathcal{L}$ is the likelihood, and $P(\mathcal{D} \mid \mathcal{H}, \mathcal{I})$ is the model evidence. As said before, we use MCMC techniques to explore the posterior in Eq. (4). We discuss below our choices for the priors and the likelihood function

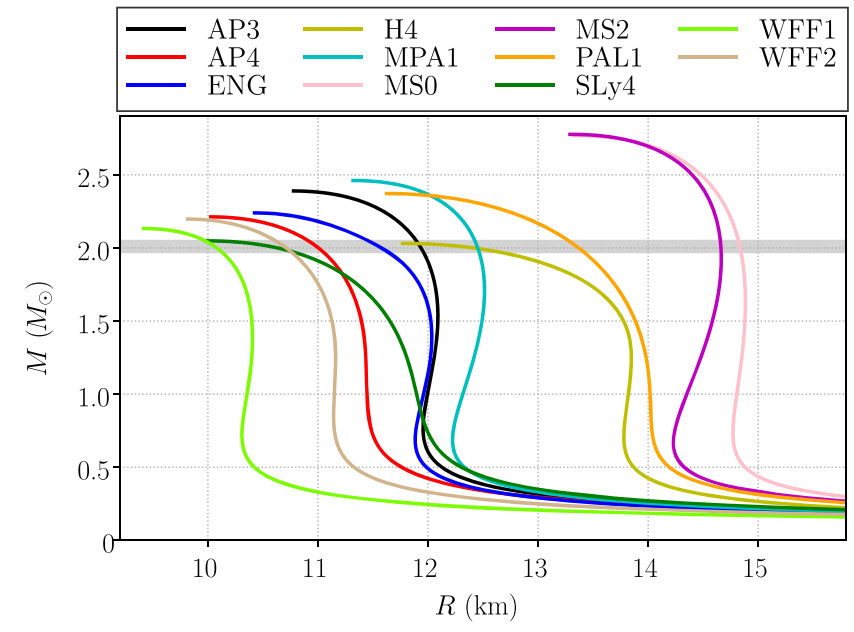

FIG. 2. The mass-radius relation of NSs (in GR) for the 11 EOSs that are adopted in the study. The mass constraint (with $1 \sigma$ uncertainty) from PSR J0348 + 0432 [35] is depicted in grey. The color coding for different EOSs is kept consistent for all figures in this paper. [see Eq. (9)]. We assume that observations with different binary pulsars are independent.

We now explain how we employ the MCMC technique to get the posterior by combining binary pulsar systems. Let us assume that $N$ pulsars $(N=1,2,5$, see below $)$ are used to constrain the $\left(\alpha_{0}, \beta_{0}\right)$ parameter space. To obtain a complete description of the gravitational dipolar radiation of these systems in the DEF theory, we need $N+2$ free parameters in the MCMC runs, which are $\boldsymbol{\theta}=\left\{\alpha_{0}, \beta_{0}, \tilde{\rho}_{c}^{(i)}\right\}$, where $\tilde{\rho}_{c}^{(i)}(i=1, \ldots, N)$ is the Jordanframe central matter density of pulsar $i$ [61]. As an initial value to the TOV solver, we also need the value of the scalar field in the center of a NS, $\varphi_{c}^{(i)}$, but the latter is fixed iteratively by requiring that all pulsars have a common asymptotic value of $\varphi, \varphi_{0} \equiv \alpha_{0} / \beta_{0}$. Given $\tilde{\rho}_{c}^{(i)}$ and $\varphi_{c}^{(i)}$ for pulsar $i$, we integrate the modified TOV equations [see Eq. (7) in Ref. [14] or Eq. (3.6) in Ref. [15]] with initial conditions given by Eq. (3.14) in Ref. [15]. During the integration, we use tabulated data of EOSs and linearly interpolate them in the logarithmic space of the matter density $\tilde{\rho}$, the pressure $\tilde{p}$, and the number density $\tilde{n}$ [55]. Note that only one quantity among $\{\tilde{\rho}, \tilde{p}, \tilde{n}\}$ is free, while the others are determined by the EOS. The end products of the integration provide us with, for each pulsar, the gravitational mass $m_{A}^{(i)}$, the baryonic mass $\bar{m}_{A}^{(i)}$, the NS radius $R^{(i)}$, and the effective scalar coupling $\alpha_{A}^{(i)}$ [15].

For the MCMC runs, we use a uniform prior on $\log _{10}\left|\alpha_{0}\right|$ for $\left|\alpha_{0}\right| \in\left[5 \times 10^{-6}, 3.4 \times 10^{-3}\right]$, where $3.4 \times 10^{-3}$ is the limit obtained from the Cassini spacecraft $[29,62]$. We pick the parameter $\beta_{0}$ uniformly in the range $[-5,-4]$, which corresponds to a sufficiently large parameter space where the scalarization phenomena can take place [14,24]. During the exploration of the parameter space, we restrict the values of $\left(\alpha_{0}, \beta_{0}\right)$ to this rectangle region, as well, in order to avoid overusing computational time in uninteresting 
regions. We choose the initial central matter densities $\left\{\tilde{\rho}_{c}^{(i)}\right\}$ around their GR values, but they are allowed to explore a very large range in the simulations. As we discuss below, we perform convergence tests and verify that when evolving the chains, all parameters in $\boldsymbol{\theta}$ quickly lose memory of their initial values.

During the MCMC runs, we evolve the $N+2$ free parameters according to an affine-invariant ensemble sampler, which was implemented in the emcee package [63-65]. At every step, we solve the $N$ sets of modified TOV equations on the fly, using the values listed in Table I [66] for the companion masses of the binary pulsars.

Then, for the decay of the binary's orbital period, which enters the likelihood function [see Eq. (9)], we use the dipolar contribution from the scalar field and the quadrupolar contribution from the tensor field as given by the following well-known formulas $[7,67]$ :

$$
\begin{aligned}
& \dot{P}_{b}^{\text {dipole }}=-\frac{2 \pi G_{*}}{c^{3}} g(e)\left(\frac{2 \pi}{P_{b}}\right) \frac{m_{p} m_{c}}{m_{p}+m_{c}}\left(\alpha_{A}-\alpha_{0}\right)^{2}, \\
& \dot{P}_{b}^{\text {quad }}=-\frac{192 \pi G_{*}^{5 / 3}}{5 c^{5}} f(e)\left(\frac{2 \pi}{P_{b}}\right)^{5 / 3} \frac{m_{p} m_{c}}{\left(m_{p}+m_{c}\right)^{1 / 3}},
\end{aligned}
$$

with

$$
\begin{gathered}
g(e) \equiv\left(1+\frac{e^{2}}{2}\right)\left(1-e^{2}\right)^{-5 / 2}, \\
f(e) \equiv\left(1+\frac{73}{24} e^{2}+\frac{37}{96} e^{4}\right)\left(1-e^{2}\right)^{-7 / 2} .
\end{gathered}
$$

We find that higher-order terms, as well as the subdominant scalar quadrupolar radiation, give negligible contributions to this study. Notice that in Eq. (5), we have replaced the effective scalar coupling of the WD companion with the linear matter-scalar coupling constant since, for a weakly self-gravitating WD, $\alpha_{A} \simeq \alpha_{0}$ in the $\beta_{0}$ range of interest [68]. Furthermore, we can approximate the bare gravitational constant $G_{*}$ in the above equations with the Newtonian gravitational constant $G_{N}=G_{*}\left(1+\alpha_{0}^{2}\right)$ since $\left|\alpha_{0}\right| \ll 1$ (e.g., from the Cassini spacecraft $[29,62]$ ).

We construct the logarithmic likelihood for the MCMC runs as

$\ln \mathcal{L} \propto-\frac{1}{2} \sum_{i=1}^{N}\left[\left(\frac{\dot{P}_{b}^{\mathrm{int}}-\dot{P}_{b}^{\mathrm{th}}}{\sigma_{\dot{P}_{b}}^{\mathrm{obs}}}\right)^{2}+\left(\frac{m_{p} / m_{c}-q}{\sigma_{q}^{\mathrm{obs}}}\right)^{2}\right]$,

where for PSRs J1909-3744 and J2222 - 0137, we replace the second term in the squared brackets with $\left[\left(m_{p}-m_{p}^{\mathrm{obs}}\right) / \sigma_{m_{p}}^{\mathrm{obs}}\right]^{2}$. In Eq. (9), the predicted orbital decay from the theory is $\dot{P}_{b}^{\text {th }} \equiv \dot{P}_{b}^{\text {dipole }}+\dot{P}_{b}^{\text {quad }}$, and $\sigma_{X}^{\text {obs }}$ is the observational uncertainty for $X \in\left\{\dot{P}_{b}^{\text {int }}, q, m_{p}\right\}$, as given in
Table I. Note that $\dot{P}_{b}^{\text {th }}$ and $m_{p}$ implicitly depend on $\left(\alpha_{0}, \beta_{0}, \boldsymbol{\Xi}\right)$, through direct integration of TOV equations in the DEF theory.

For each EOS, we perform four separate MCMC runs:

(i) 1 pulsar: PSR J0348 + 0432 (J0348),

(ii) 1 pulsar: PSR J1738 + 0333 (J1738),

(iii) combining 2 pulsars: PSRs J0348 +0432 and $\mathrm{J} 1738+0333$ (2PSRs),

(iv) combining 5 pulsars: PSRs J0348 + 0432, $\mathrm{J} 1012+5307, \mathrm{~J} 1738+0333, \mathrm{~J} 1909-3744$, and J2222 - 0137 (5PSRs).

We pick $J 0348$ and $\mathrm{J} 1738$ because of their mass difference (2.01 $M_{\odot}$ and $1.46 M_{\odot}$, respectively) and their high timing precision (see Table I), which leads to interesting differences in the constraints on the DEF parameters, especially on $\beta_{0}$. For each run, we accumulate sufficient MCMC samples to guarantee the convergence of MCMC runs. By using the Gelman-Rubin statistic [69], we find that, for each EOS, 200000 samples for cases J0348 and J1738, and 400000 samples for cases 2PSRs and 5PSRs are enough, respectively. We discard the first half chain points of these 44 runs (4cases $\times 11 \mathrm{EOSs}$ ) as the BURN-IN phase [64,70], while we use the remaining samples to hypothesize on the parameters $\left(\alpha_{0}, \beta_{0}\right)$.

As an example, we show in Fig. 3 the marginalized 2D distribution in the parameter space of $\left(\log _{10}\left|\alpha_{0}\right|,-\beta_{0}\right)$ for the case 5PSRs and the EOS SLy4. As mentioned above, we distribute the initial values of $\log _{10}\left|\alpha_{0}\right|$ and $-\beta_{0}$ uniformly in the rectangle region of Fig. 3. We see that after MCMC simulations, the region with large $\left|\alpha_{0}\right|$ or large (negative) $\beta_{0}$

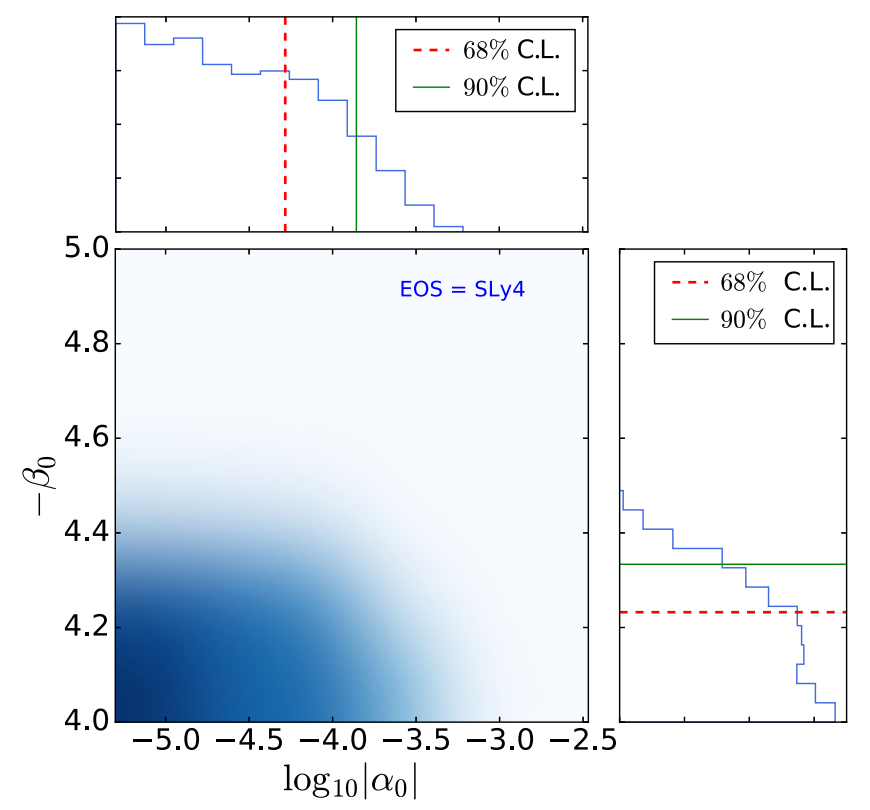

FIG. 3. The marginalized 2D distribution of $\left(\log _{10}\left|\alpha_{0}\right|,-\beta_{0}\right)$ from MCMC runs on the five pulsars listed in Table I, for the EOS SLy4. The marginalized 1D distributions and the extraction of upper limits are illustrated in the upper and right panels. 


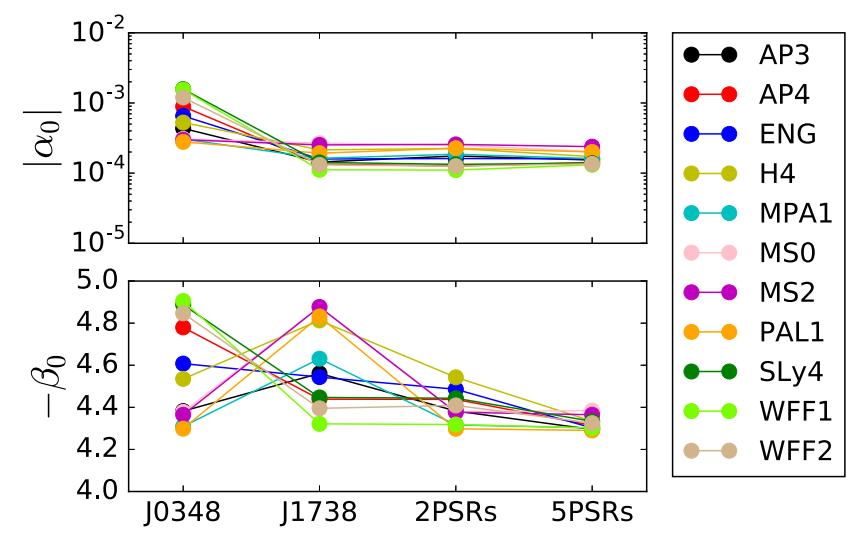

FIG. 4. Marginalized upper limits on $\left|\alpha_{0}\right|$ (upper panel) and $-\beta_{0}$ (lower panel) at 90\% C.L. These limits are obtained from PSRs $\mathrm{J} 0348+0432$ (J0348), J1738 + 0333 (J1738), a combination of them (2PSRs), and a combination of PSRs J0348 + 0432, $\mathrm{J} 1012+5307, \mathrm{~J} 1738+0333, \mathrm{~J} 1909-3744$, and $\mathrm{J} 2222-$ 0137 (5PSRs).

is no longer populated, and only a small corner is consistent with the observational results of the five NS-WD binary pulsars.

Furthermore, we extract the upper limits of $\log _{10}\left|\alpha_{0}\right|$ and $-\beta_{0}$ from their marginalized 1D distributions at $68 \%$ and $90 \%$ C.L.s. We summarize the upper limits at $90 \%$ C.L. from all 44 runs in Fig. 4. It is interesting to observe the following facts. First, for all EOSs, J1738 gives a more constraining limit on $\alpha_{0}$ than J0348. This result might be due to the fact that the $\sigma_{\dot{P}_{b}}^{\text {obs }}$ of $J 1738$ is about 2 orders of magnitude smaller than that of J0348, thus giving a tighter limit on $\alpha_{0}^{2}$ by roughly the same order of magnitude. Second, the constraints on $\beta_{0}$ from J0348 and J1738 are extremely EOS dependent. This should be a consequence of the masses of the NSs, which are (in GR) $1.46 M_{\odot}$ for $\mathrm{J} 1738$, and $2.01 M_{\odot}$ for J0348. For EOSs that favor spontaneous scalarization at around $1.4-1.5 M_{\odot}$, J1738 gives a better limit, while for EOSs that favor spontaneous scalarization at around $2 M_{\odot}$, J0348 gives a better limit. This trend is also consistent with Fig. 5 (to be introduced below). Third, by combining two pulsars (2PSRs), NSs are limited to scalarize at neither $1.4-1.5 M_{\odot}$ nor $\sim 2 M_{\odot}$. Therefore, almost for all EOSs, $\beta_{0}$ is well constrained. This result demonstrates the power of properly using multiple pulsars with different NS masses to constrain the DEF parameter space for any EOS. Fourth, we obtain the most stringent constraints with five pulsars (5PSRs). This is especially true for $\beta_{0}$, which is constrained at the level of about -4.2 (68\% C.L.) and about -4.3 (90\% C.L.) for all EOSs. Finally, we list in Table II the marginalized 1D limits for 5PSRs. We use them in the next section when combining binary pulsars with laser-interferometer GW observations.

Considering the results that we have obtained when combining the five pulsars (5PSRs), one might wonder

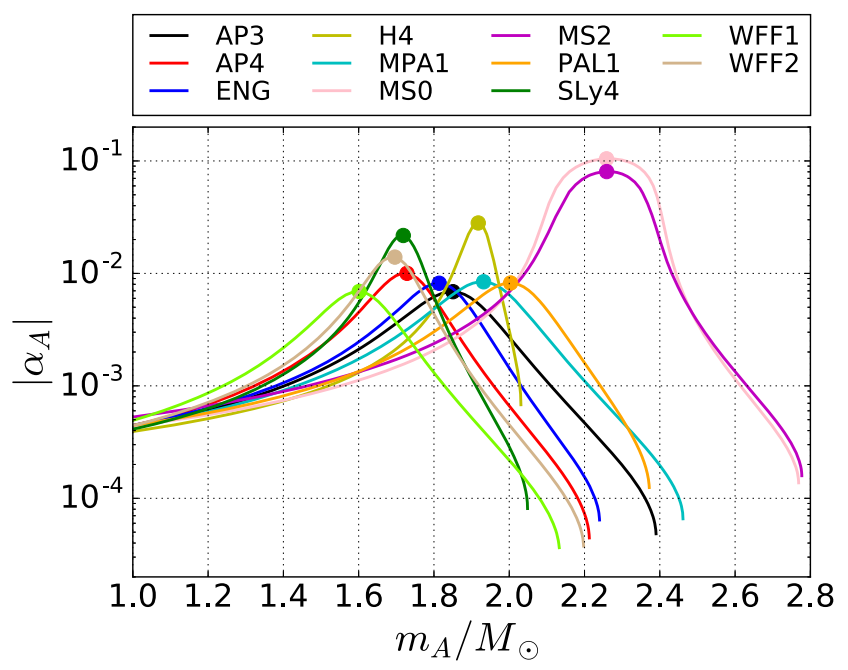

FIG. 5. The effective scalar coupling $\left|\alpha_{A}\right|$ that an isolated NS could still develop after taking into account the 95\% C.L. constraints from the five pulsars (see Table II). The point of the maximum $\left|\alpha_{A}\right|$ is marked with a dot, and the values (and the corresponding masses) are listed in Table II.

whether isolated NSs can still be strongly scalarized. To address this question, we use the limits on $\left(\alpha_{0}, \beta_{0}\right)$ and calculate the effective scalar coupling that a NS can still develop as a function of the NS mass, for the 11 EOSs used in this paper. The results at $90 \%$ C.L. are summarized in Fig. 5, while in Table II, we list the maximally allowed effective scalar couplings at $68 \%$ and $90 \%$ C.L.s, and their corresponding (gravitational) NS masses (marked as dots in Fig. 5).

Figure 5 clearly shows the nonperturbative nature of the scalarization phenomenon. The (absolute values of the) maximally allowed effective scalar coupling for NSs can be as large as $\mathcal{O}\left(10^{-2}\right)$ and even 0.1 if the limits at $90 \%$ C.L. are used, while those values are $\lesssim 10^{-3}$ if one uses the limits at 68\% C.L. (not shown in Fig. 5, but listed in Table II). Furthermore, quite remarkably, Fig. 5 shows that there are scalarization windows (this feature could also be seen in Fig. 1 for the EOS SLy4). What we mean is that the NS masses for the five most constraining pulsars are $1.46 M_{\odot}$ (PSR J1738 + 0333), $1.54 M_{\odot}$ (PSR J1909-3744), $1.76 M_{\odot}$ (PSR J2222-0137), $1.83 M_{\odot}$ (PSR $1012+5307$ ), and $2.01 M_{\odot}$ (PSR J0348 + 0432). For these specific masses, using the 11 EOSs that can give rise to spontaneous scalarization, we have stringently constrained the DEF parameters. However, some EOSs can still allow NSs to scalarize strongly (i.e., acquire large effective scalar couplings) for other values of the masses. As Fig. 5 shows, using limits at $90 \%$ C.L., NSs with EOSs AP4, SLy4, and WFF2 can still have $\left|\alpha_{A}\right| \gtrsim \mathcal{O}\left(10^{-2}\right)$ if the NS masses are in the range $m_{A}=1.70-1.73 M_{\odot}$, NSs with EOS $\mathrm{H} 4$ can still be scalarized to $\left|\alpha_{A}\right| \sim 0.03$ with $m_{A} \simeq 1.92 M_{\odot}$, and NSs with EOSs MS0 and MS2 can still be strongly scalarized to $\left|\alpha_{A}\right| \simeq 0.1$ with $m_{A} \simeq 2.26 M_{\odot}$. 
TABLE II. Limits on the parameters of the massless mono-scalar-tensor DEF theory for different EOSs when applying the MCMC analysis to the five pulsars $\mathrm{J} 0348+0432$, J1012 + 5307, J1738 + 0333, J1909 - 3744, and J2222 - 0137 . Results at $68 \%$ and 90\% C.L.s are listed. Here, $\left|\alpha_{A}\right|^{\max }$ is the maximum effective scalar coupling that a NS could still possess without violating the limits, and $m_{A}^{\max }$ is the corresponding (gravitational) mass at this maximum effective scalar coupling (see Fig. 5).

\begin{tabular}{|c|c|c|c|c|c|c|c|c|}
\hline \multirow[b]{2}{*}{ EOS } & \multicolumn{4}{|c|}{$68 \%$ confidence level } & \multicolumn{4}{|c|}{$90 \%$ confidence level } \\
\hline & $\left|\alpha_{0}\right|$ & $-\beta_{0}$ & $m_{A}^{\max } / M_{\odot}$ & $\left|\alpha_{A}\right|^{\max }$ & $\left|\alpha_{0}\right|$ & $-\beta_{0}$ & $m_{A}^{\max } / M_{\odot}$ & $\left|\alpha_{A}\right|^{\max }$ \\
\hline AP3 & $6.5 \times 10^{-5}$ & 4.21 & 1.83 & $1.1 \times 10^{-3}$ & $1.5 \times 10^{-4}$ & 4.29 & 1.85 & $6.9 \times 10^{-3}$ \\
\hline AP4 & $5.5 \times 10^{-5}$ & 4.24 & 1.71 & $1.2 \times 10^{-3}$ & $1.4 \times 10^{-4}$ & 4.31 & 1.73 & $1.0 \times 10^{-2}$ \\
\hline ENG & $6.0 \times 10^{-5}$ & 4.21 & 1.80 & $1.0 \times 10^{-3}$ & $1.6 \times 10^{-4}$ & 4.30 & 1.81 & $8.2 \times 10^{-3}$ \\
\hline $\mathrm{H} 4$ & $5.7 \times 10^{-5}$ & 4.24 & 1.91 & $1.3 \times 10^{-3}$ & $1.7 \times 10^{-4}$ & 4.33 & 1.92 & $2.8 \times 10^{-2}$ \\
\hline MPA1 & $5.7 \times 10^{-5}$ & 4.22 & 1.92 & $1.1 \times 10^{-3}$ & $1.6 \times 10^{-4}$ & 4.30 & 1.93 & $8.4 \times 10^{-3}$ \\
\hline MSO & $7.7 \times 10^{-5}$ & 4.28 & 2.26 & $2.7 \times 10^{-3}$ & $2.0 \times 10^{-4}$ & 4.38 & 2.26 & $1.0 \times 10^{-1}$ \\
\hline MS2 & $7.9 \times 10^{-5}$ & 4.26 & 2.24 & $2.1 \times 10^{-3}$ & $2.4 \times 10^{-4}$ & 4.36 & 2.26 & $8.0 \times 10^{-2}$ \\
\hline PAL1 & $7.3 \times 10^{-5}$ & 4.21 & 1.99 & $1.2 \times 10^{-3}$ & $2.0 \times 10^{-4}$ & 4.29 & 2.00 & $8.2 \times 10^{-3}$ \\
\hline SLy4 & $5.2 \times 10^{-5}$ & 4.23 & 1.71 & $1.1 \times 10^{-3}$ & $1.4 \times 10^{-4}$ & 4.33 & 1.72 & $2.2 \times 10^{-2}$ \\
\hline WFF1 & $5.3 \times 10^{-5}$ & 4.21 & 1.58 & $9.1 \times 10^{-4}$ & $1.3 \times 10^{-4}$ & 4.30 & 1.60 & $6.9 \times 10^{-3}$ \\
\hline WFF2 & $5.5 \times 10^{-5}$ & 4.24 & 1.68 & $1.2 \times 10^{-3}$ & $1.4 \times 10^{-4}$ & 4.32 & 1.70 & $1.4 \times 10^{-2}$ \\
\hline
\end{tabular}

Those scalarization windows could be closed in the future if binary pulsars with these masses are discovered and their gravitational dipolar radiation is constrained by pulsar timing. As we discuss in Sec. IV, the presence of scalarization windows also opens the interesting possibility to close these gaps with future GW observations from BNSs, if the masses of the NSs lie in the scalarization window.

\section{PROJECTED SENSITIVITIES FOR LASER-INTERFEROMETER GRAVITATIONAL-WAVE DETECTORS}

Having determined constraints on the DEF's parameter space from binary pulsars (see Table II) and having found scalarization windows, we now address the question of whether present and future laser-interferometer GW observations on the ground can still improve these limits and close the gaps. Two scenarios are considered: (i) asymmetric BNS systems, equipped with separation-independent effective scalar couplings, whose gravitational dipolar radiation during the inspiral can modify the GW phasing [16,71], and (ii) BNS systems that dynamically develop scalarization during the late stage of the inspiral, leading to significant, nonperturbative changes in the GW signal $[20,24,26,27]$. A complete description of BNSs in the DEF theory should include both effects. However, complete waveform models from the theory are still not available, so here we investigate the two scenarios separately to obtain some conservative understanding of the whole picture.

\section{A. Dipole radiation for binary neutron-star inspirals}

The presence of a scalar field can significantly modify the inspiral of an asymmetric BNS system because of the additional energy radiated off by the scalar d.o.f. The most prominent effect is a modification of the phase evolution in GW signals. For two NSs with effective scalar couplings $\alpha_{A}$ and $\alpha_{B}$, respectively, one finds for the evolution of the orbital frequency $\Omega$, up to $2.5 \mathrm{PN}$ order [71-73],

$$
\frac{\dot{\Omega}}{\Omega^{2}}=\frac{\eta}{1+\alpha_{A} \alpha_{B}}\left[(\Delta \alpha)^{2} \mathcal{V}^{3}+\frac{96}{5} \kappa \mathcal{V}^{5}+\mathcal{O}\left(\mathcal{V}^{6}\right)\right],
$$

where $\Delta \alpha \equiv \alpha_{A}-\alpha_{B}, \eta \equiv m_{A} m_{B} / M^{2}$, and the (dimensionless) "characteristic" velocity

$$
\mathcal{V} \equiv\left[G_{*}\left(1+\alpha_{A} \alpha_{B}\right) M \Omega\right]^{1 / 3} / c .
$$

The quantity $\kappa$ is given in Refs. [7,35]. In GR, one has $\alpha_{A}=\alpha_{B}=0$ and $\kappa=1$. Note that there is also a subdominant contribution from the scalar quadrupolar waves at 2.5 PN order, which, however, can be absorbed by a $\lesssim 1 \%$ change in the mass parameters. Here, we assume that $\alpha_{A}$ and $\alpha_{B}$ are constant during the inspiral, and their values are obtained from isolated NSs. This assumption is valid as long as the induced or dynamical scalarization mechanisms are not triggered.

For an asymmetric compact binary where $\alpha_{A} \neq \alpha_{B}$, the most prominent deviation from the GR phase evolution is determined by the dipole term in Eq. (10), i.e., the contribution $\propto \mathcal{V}^{3}$. To leading order, the offset in the number of GW cycles in band until merger due to the dipole term is given by

$$
\Delta \mathcal{N}_{\text {dipole }} \simeq-\frac{25}{21504 \pi} \eta^{-1} \mathcal{V}_{\mathrm{in}}^{-7}(\Delta \alpha)^{2},
$$

where $\mathcal{V}_{\text {in }}$ corresponds to $\mathcal{V}$ in Eq. (11) when the merging system enters the band of the GW detector, i.e., when $\Omega=\pi f_{\text {in }}$ (see Refs. [16,71] for details). In the above 
TABLE III. The number of GW cycles in GR, $\mathcal{N}_{\mathrm{GR}}$, for a BNS merger with masses $\left(1.25 M_{\odot}, 1.7 M_{\odot}\right)$ for frequencies $f>f_{\text {in }}$, and its change due to the dipole radiation in the DEF's theory, $\Delta \mathcal{N}_{\text {dipole }}$, assuming $|\Delta \alpha| \equiv\left|\alpha_{A}-\alpha_{B}\right| \simeq 0.0199$, which comes from the maximally allowed effective scalar couplings for the EOS SLy4 at 90\% C.L. (see Fig. 5). The limits on the contributions from leadingorder spin-orbit and spin-spin terms, $\left|\Delta \mathcal{N}_{\beta}\right|$ and $\left|\Delta \mathcal{N}_{\sigma}\right|$, are listed where the (dimensionless) spins of the double pulsar (when it merges in $86 \mathrm{Myr}$ ) are used. For $\left|\Delta \mathcal{N}_{\beta}\right|$ and $\left|\Delta \mathcal{N}_{\sigma}\right|$, we also show parenthetically the case where both NSs are spinning at the maximal spin that we have ever observed (in an eclipsing binary pulsar J1748 - 2446ad).

\begin{tabular}{lccccc}
\hline \hline Detector & $f_{\text {in }}(\mathrm{Hz})$ & $\mathcal{N}_{\text {GR }}$ & $\Delta \mathcal{N}_{\text {dipole }}$ & $\left|\Delta \mathcal{N}_{\beta}\right|$ & $\left|\Delta \mathcal{N}_{\sigma}\right|$ \\
\hline aLIGO & 10 & $1.5 \times 10^{4}$ & $-3.7 \times 10^{1}$ & $<0.76\left(<3.5 \times 10^{1}\right)$ & $<1.8 \times 10^{-6}(<0.43)$ \\
CE & 5 & $4.8 \times 10^{4}$ & $-1.9 \times 10^{2}$ & $<1.2\left(<5.6 \times 10^{1}\right)$ & $<2.3 \times 10^{-6}(<0.55)$ \\
ET & 1 & $7.0 \times 10^{5}$ & $-8.1 \times 10^{3}$ & $<3.5\left(<1.6 \times 10^{2}\right)$ & $<3.9 \times 10^{-6}(<0.93)$ \\
\hline \hline
\end{tabular}

equation, we have used the approximation $\kappa \simeq 1$ and the fact that $\mathcal{V}_{\text {in }}$ is much smaller than $\mathcal{V}$ just before merger. Within the approximation of Eq. (12), one can use $\mathcal{V}_{\text {in }} \simeq\left(G_{\mathrm{N}} M \pi f_{\text {in }}\right)^{1 / 3} / c$, i.e., replacing the effective gravitational constant $G_{*}\left(1+\alpha_{A} \alpha_{B}\right)$ in Eq. (11) by the Newtonian gravitational constant $G_{\mathrm{N}} \equiv G_{*}\left(1+\alpha_{0}^{2}\right)$ [7,15]. Again, we stress that Eq. (12) is based on the assumption that the effective scalar couplings of the two NSs, $\alpha_{A}$ and $\alpha_{B}$, remain unchanged during the inspiral in the detector's sensitive frequency band. It therefore neglects the phenomenon of induced scalarization, which can occur in a BNS system, when the unscalarized NS is sufficiently exposed to the scalar field of the scalarized companion [25]. This can reduce the dipolar radiation considerably on short ranges if $\alpha_{A}$ approaches $\alpha_{B}$, and it can lead to a characteristic change in the late phase evolution of the merging BNSs. Dynamically changing effective scalar couplings are explored in the next subsection.

To obtain a rough understanding of the effects of dipolar radiation, let us calculate the dephasing from GR by an asymmetric BNS inspiral with $m_{A}=1.25 M_{\odot}$ and $m_{B}=1.7 M_{\odot}$. According to Fig. 5, at present, binarypulsar experiments cannot exclude $\left|\alpha_{A}\right|$ as large as $10^{-2}-10^{-1}$ for NSs of a certain mass range, which depends on the EOS. For the EOS SLy4, we find from the corresponding (dark green) curve in Fig. 5, $\left|\alpha_{A}\right| \simeq$ 0.0007 and $\left|\alpha_{B}\right| \simeq 0.0206$; hence, $|\Delta \alpha| \equiv\left|\alpha_{A}-\alpha_{B}\right| \simeq$ 0.0199 .

In our study, we consider the Advanced LIGO (aLIGO) detectors at design sensitivity [28], and future groundbased detectors, such as the Cosmic Explorer (CE) and the Einstein Telescope (ET). We use the starting frequencies $f_{\text {in }}=10 \mathrm{~Hz}$ for aLIGO, $f_{\text {in }}=5 \mathrm{~Hz}$ for $\mathrm{CE}$, and $f_{\text {in }}=$ $1 \mathrm{~Hz}$ for ET $[23,74,75]$. In Table III, we list the number of GW cycles as predicted by GR, $\mathcal{N}_{\mathrm{GR}}$, and the change in the number of cycles caused by the existence of a dipole radiation for a BNS signal, $\Delta \mathcal{N}_{\text {dipole. }}$ From Table III, we can already see that, given the BNS parameters, current bounds by pulsars still leave room for significant timedomain phasing modifications in BNS mergers, in particular, if one of the NSs falls into the scalarization window of about $1.7 M_{\odot}$ (for EOSs AP4, SLy4, and WFF2) to $1.9 M_{\odot}$ (for the EOS H4), or if one NS's mass significantly exceeds $2 M_{\odot}$ (for EOSs MSO and MS2). As reference points, we also list in Table III the changes in the number of GW cycles from spin-orbit and spin-spin effects. Indeed, from the leading-order spin-orbit (1.5 PN) and spin-spin (2 PN) contributions to the GW phasing $[18,76]$, one has

$$
\begin{gathered}
\Delta \mathcal{N}_{\beta} \simeq \frac{5}{64 \pi} \eta^{-1} \mathcal{V}_{\text {in }}^{-2} \beta, \\
\Delta \mathcal{N}_{\sigma} \simeq-\frac{5}{32 \pi} \eta^{-1} \mathcal{V}_{\text {in }}^{-1} \sigma,
\end{gathered}
$$

where

$$
\begin{gathered}
\beta=\frac{1}{12} \sum_{i=A, B}\left(113 \frac{m_{i}^{2}}{M^{2}}+75 \eta\right) \hat{\boldsymbol{L}} \cdot \chi_{i}, \\
\sigma=\frac{\eta}{48}\left[-247 \chi_{A} \cdot \chi_{B}+721\left(\hat{\boldsymbol{L}} \cdot \chi_{A}\right)\left(\hat{\boldsymbol{L}} \cdot \chi_{B}\right)\right] .
\end{gathered}
$$

The (dimensionless) spins of a BNS system, $\chi_{A}$ and $\chi_{B}$, are likely to be small in magnitude. The parameters $\beta$ and $\sigma$ are maximized when two spins are aligned with the direction of the orbital angular momentum, $\hat{L}$. The limits on $\left|\Delta \mathcal{N}_{\beta}\right|$ and $\left|\Delta \mathcal{N}_{\sigma}\right|$ are listed in Table III, where we have used the (dimensionless) spins of the double pulsar system, which is the only double NS system where two spins are precisely measured. When the double pulsar evolves to the time of its merger 86 Myr from now, one has $\left|\chi_{A}\right| \simeq 0.014$ and $\left|\chi_{B}\right| \simeq 0.00002$ [77], assuming a canonical moment of inertia $10^{38} \mathrm{~kg} \mathrm{~m}^{2}$ for NSs. As we can see from Table III, if the spins of BNSs to be discovered by GW detectors are comparable to that of the double pulsar, the inclusion of spins only affects the number of GW cycles at the percentage level at most. In addition, because groundbased detectors could observe BNSs from a population different from the one observed with pulsar timing, we also give $\left|\Delta \mathcal{N}_{\beta}\right|$ and $\left|\Delta \mathcal{N}_{\sigma}\right|$ in Table III when the (dimensionless) spin of the fastest rotating pulsar ever observed, PSR J1748 - 2446ad ( $P=1.4 \mathrm{~ms}$ ) [78], is used for both NSs [79]. Even in this extreme case with $\left|\chi_{A}\right|=\left|\chi_{B}\right| \simeq 0.26$ 
(assuming a canonical mass $\left.1.4 M_{\odot}\right),\left|\Delta \mathcal{N}_{\text {dipole }}\right|$ is still larger (or comparable to, in the case of the Advanced LIGO) than the upper limits of $\left|\Delta \mathcal{N}_{\beta}\right|$ and $\left|\Delta \mathcal{N}_{\sigma}\right|$.

The dephasing quantity $\Delta \mathcal{N}_{\text {dipole }}$ is nevertheless a crude indicator for realistic detectability. In reality, one has to consider various degeneracies between binary parameters, the waveform templates that are used for detection and parameter estimation, the power spectral density (PSD) of noises in GW detectors, $S_{n}(f)$, the signal-to-noise ratio (SNR) of an event $\rho$, and so on. In order to obtain more quantitative estimates of the constraints on dipolar radiation that can be expected from GW detectors, one would need to compute Bayes factors between two alternatives [80] or apply cutting-edge parameter-estimation techniques, for example, the MCMC or nested sampling [81]. However, given the limited scope of our analysis, for simplicity, here, we adopt the Fisher-matrix approach [17,71,82,83], although we are aware of the fact that for events with mild SNR $(\rho \sim 10)$, the Fisher matrix can have pitfalls [84]. In Appendix A, we review the main Fisher-matrix tools that we use.

In Fig. 6, we summarize their dimensionless noise spectral density $\sqrt{f S_{n}(f)}[23,74,75]$ and also show a hypothetical BNS signal. For all the studies, we fix the luminosity distance to $D_{L}=200 \mathrm{Mpc}$ for aLIGO, CE, and ET. Indeed, within such a distance, aLIGO alone is supposed to observe 0.2-200 BNS events annually at design sensitivity [85]. With the four-site network incorporating LIGO-India at design sensitivity, the number of detectable BNS events will double [85]. Therefore, it is a realistic setting to discuss BNS events for aLIGO; to be conservative, we only consider a two-detector network for aLIGO in our study. CE and ET have better sensitivities and

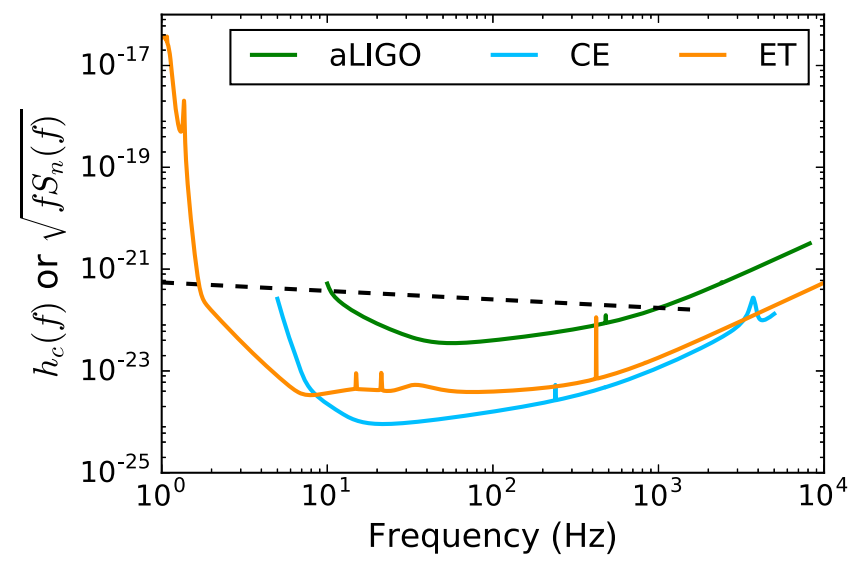

FIG. 6. Dimensionless noise spectral density $\sqrt{f S_{n}(f)}$ of aLIGO, CE, and ET GW detectors. The quantity $S_{n}(f)$ is the one-side design PSD [23,74,75]. The dashed line describes the (dimensionless) pattern-averaged characteristic strain $h_{c}(f) \equiv$ $2 f|\tilde{h}(f)|$ for a BNS with rest-frame masses $\left(1.25 M_{\odot}, 1.63 M_{\odot}\right)$ at $200 \mathrm{Mpc}(z \simeq 0.0438)$, up to the innermost-stable circular orbit given by Eq. (A3). thus will have larger SNRs for these events; besides, they will be able to detect a larger number of BNSs, including those with unfavorable orientations. Using the standard cosmological model [86], the redshift associated with $D_{L}=200 \mathrm{Mpc}$ is $z \simeq 0.0438$, and we take it into account in our Fisher-matrix calculation, even if it generates a small effect. Moreover, we always report masses in the rest frame of a BNS system.

The Fisher matrix is constructed as usual from the Fourier-domain waveform $\tilde{h}(f)$ [71,82,83],

$$
\Gamma_{a b} \equiv\left(\partial_{a} \tilde{h}(f) \mid \partial_{b} \tilde{h}(f)\right),
$$

with $\partial_{a} \tilde{h}(f)$ being the partial derivative of the parameter labeled " $a$ " (see Appendix A for definitions and notations). We use the waveform parameters $\left\{\ln \mathcal{A}, \ln \eta, \ln \mathcal{M}, t_{c}\right.$, $\left.\Phi_{c},(\Delta \alpha)^{2}\right\}$ to construct the $6 \times 6$ Fisher matrix $\Gamma_{a b}$. The inverse of the Fisher matrix is the correlation matrix for these parameters, from which we can read their uncertainties and correlations $[17,71,82,83]$.

In Fig. 7, we plot with dashed lines the uncertainties in $|\Delta \alpha|$ obtained with three GW detectors (aLIGO, CE, and ET) for an asymmetric BNS with rest-frame masses $m_{A}=$ $1.25 M_{\odot}$ and $m_{B}>1.25 M_{\odot}$, located at $D_{L}=200 \mathrm{Mpc}$. For a BNS of masses, for example, $\left(1.25 M_{\odot}, 1.63 M_{\odot}\right)$,

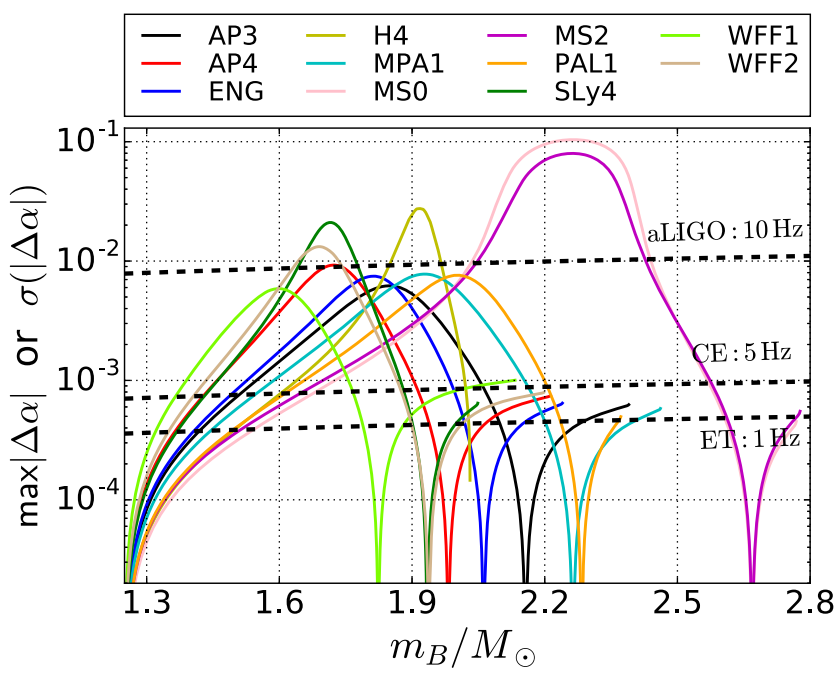

FIG. 7. The sensitivities of aLIGO, CE, and ET to $|\Delta \alpha|$ [namely, the uncertainty $\sigma(|\Delta \alpha|)$ obtained from the inverse Fisher matrix] are depicted with dashed lines, as a function of $m_{B}$, for a pattern-averaged BNS inspiral signal with rest-frame component masses $\left(m_{A}=1.25 M_{\odot}, m_{B}\right)$. The starting frequencies of $\mathrm{GW}$ detectors are labeled. Luminosity distance $D_{L}=200 \mathrm{Mpc}$ is assumed. The sensitivity to $|\Delta \alpha|$ from GW detectors scales with SNR as $\rho^{-1 / 2}$. The maximum available values of $|\Delta \alpha|$ for 11 EOSs, saturating the limits from binary pulsars at $90 \%$ C.L., are shown as solid lines. If a sensitivity curve (dashed) is below a solid curve, the corresponding GW detector has the potential to improve the limit from binary pulsars for this particular EOS, with BNSs of suitable masses. 
which are the most probable masses for the newly discovered asymmetric double-NS binary pulsar PSR J1913 + 1102 [87], we find that aLIGO, CE, and ET can detect its merger at $200 \mathrm{Mpc}$ with $\rho=10.6,450$, and 153 , respectively, after averaging over pattern functions and assuming two detectors in each case. The characteristic strain of such a BNS is illustrated in the frequency domain in Fig. 6. In the large SNR limit, the uncertainties in $|\Delta \alpha|$ scale with the SNR as $\rho^{-1 / 2}$. In Fig. 8, we give the correlations between parameters obtained from the matched-filter analysis. We find that because of its low-frequency sensitivity, ET can break some degeneracy between parameters better than aLIGO and CE do.

In Fig. 7, we show with solid lines the maximum values of $|\Delta \alpha|$ at $90 \%$ C.L. from pulsars for 11 EOSs (calculated from Fig. 5). If for some NS's mass range a solid line (which is associated with a certain EOS) is above a dashed line (associated with a certain detector), then for NSs described by that EOS, the corresponding GW detector has the potential to further improve the DEF's parameters with the observation of a BNS within that mass range. From the figure, we can see that, with the expected design sensitivity curves of aLIGO, CE, and ET [23,74,75],

(i) aLIGO has the potential to further improve the current limits from binary pulsars with a discovery of a BNS of suitable masses, if the EOS of NSs is one of (or similar to) H4, MSO, MS2, SLy4, and WFF2;

(ii) $\mathrm{CE}$ and ET, because of their low-frequency sensitivity and better PSD curves, are able to significantly improve current limits from binary pulsars on the DEF's parameters, no matter what the real EOS of NSs is.

We stress that those conclusions are obtained with a Fisher-matrix analysis and should be made more robust in the future by using more sophisticated tools, notably Bayesian analysis.

\section{Constraints outside the spontaneous-scalarization regime}

With the results above, it is fairly straightforward to calculate the limits from aLIGO, CE, and ET on $\left|\alpha_{0}\right|$ when $\beta_{0}$ is outside the spontaneous scalarization regime, i.e., $\beta_{0} \gtrsim-4$, and compare them to existing limits from the Solar System and pulsars [16]. For completeness, we present the relevant results here. Shibata et al. [20] have shown that for small $\alpha_{0}$, there exists a simple relation between $\alpha_{A}, \alpha_{0}$, and $m_{A}$ as long as spontaneous scalarization does not set in [see Eq. (44) in Ref. [20]], which in our notation reads [88]

$$
\alpha_{A} \simeq \mathcal{A}_{\beta_{0}}^{(A)}\left(m_{A}, \beta_{0} ; \mathrm{EOS}\right) \alpha_{0} .
$$

With this equation at hand, one can directly convert the limits from ground-based GW detectors of Fig. 7, for any given $\beta_{0} \gtrsim-4$, into limits for $\left|\alpha_{0}\right|$ via

$$
\left|\alpha_{0}\right|=\left|\frac{\Delta \alpha}{\mathcal{A}_{\beta_{0}}^{(A)}-\mathcal{A}_{\beta_{0}}^{(B)}}\right| .
$$

Figure 9 gives the results for two different mass configurations and the EOS AP4. A more stiff EOS would generally lead to less constraining limits for ground-based GW detectors and binary pulsars. As one can see, in the range $\beta_{0} \gtrsim-4$, current Solar System and pulsar tests are already clearly more constraining than what aLIGO is expected to obtain. For CE and ET, only inspirals with a very massive component will provide constraints that are better than present limits, for a limited range of $\beta_{0}$ (see also aLIGO [89] and ET [89,90] limits from a NS-BH inspiral for the special case of $\beta_{0}=0$, i.e., Jordan-Fierz-Brans-Dicke gravity). By the time CE or ET is operational, however, the expected limits from GAIA [91] and SKA [11] will
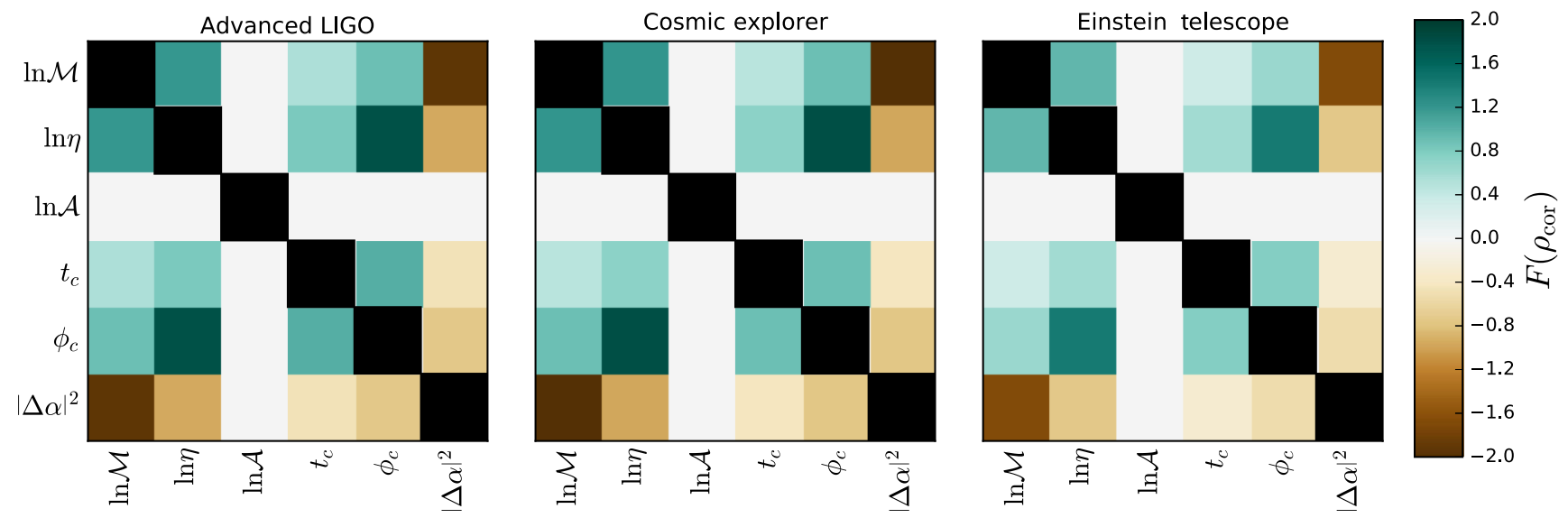

FIG. 8. The correlations between six parameters, obtained from the inverse Fisher matrix in the matched-filter analysis for a BNS with rest-frame masses $\left(1.25 M_{\odot}, 1.63 M_{\odot}\right)$. Here, $F\left(\rho_{\text {cor }}\right) \equiv \log _{10}\left[\left(1+\rho_{\text {cor }}\right) /\left(1-\rho_{\text {cor }}\right)\right]-\rho_{\text {cor }} \log _{10} 2$ is a function defined in Ref. [13] such that it counts 9's in the limit of large correlations [e.g., $F(0.99) \simeq+2, F(-0.9) \simeq-1$, and $F(0)=0$ ]. On the diagonal, $F\left(\rho_{\text {cor }}=1\right)$ diverges and is plotted in black. 


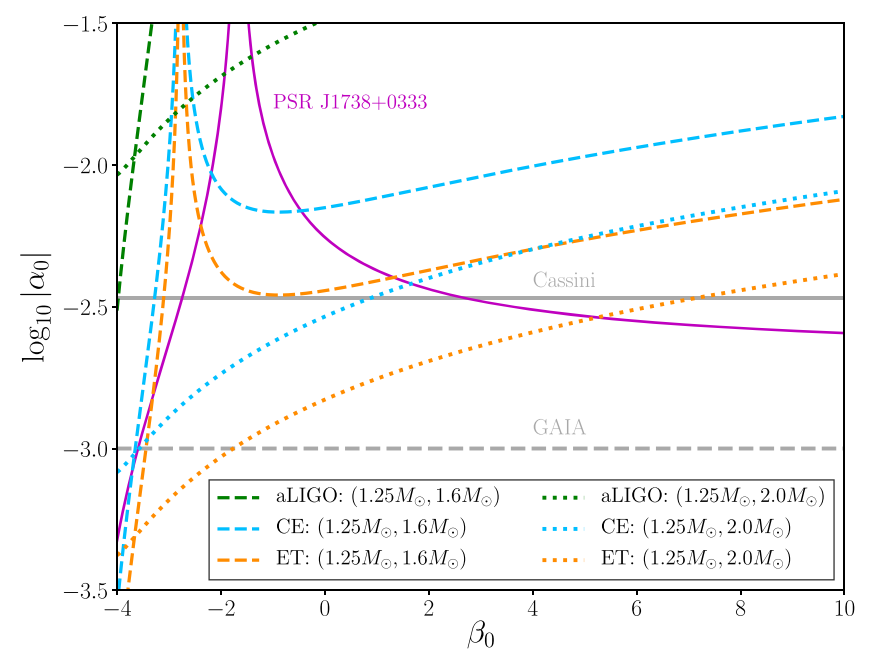

FIG. 9. Upper limits on $\left|\alpha_{0}\right|$ as a function of $\beta_{0}$ for aLIGO (green line), CE (blue line), and ET (orange line) [16]. The dashed lines correspond to a 1.25 $M_{\odot} / 1.6 M_{\odot}$ BNS merger, and the dotted lines correspond to a $1.25 M_{\odot} / 2.0 M_{\odot}$ BNS merger. The chosen EOS is AP4. For comparison, we have plotted Solar System limits (grey line) and the limits from PSR J1738 + 0333 (magenta line), which currently give the best limit for $\beta_{0} \gtrsim 3$. The limit from Cassini [62] and the limit expected from GAIA [91] are also shown.

have left little room for ground-based GW observatories in the regime. The space-based GW observatories LISA $[17,18]$ and DECIGO/BBO [19] could, in principle, also provide limits on the DEF theory from an inspiral of a NS into an intermediate-mass black hole, provided such BHs exist. However, the resulting limits on $\left|\alpha_{0}\right|$ are not expected to be better than limits from future ground-based $\mathrm{GW}$ observatories [17]. It is worth mentioning that for very large (positive) $\beta_{0}$, say, $\beta_{0} \gtrsim 10^{2}-10^{3}$, massive NSs might develop instabilities $[92,93]$, which is beyond the scope of Fig. 9.

\section{B. Dynamical scalarization}

In addition to the nonlinear gravitational selfinteraction testable with binary pulsars, GW detectors probe the nonlinear interactions between coalescing NSs. Dynamical scalarization stems from the interplay between these two regimes of strong gravity and thus offers a promising means of complementing pulsar-timing constraints on scalar-tensor theories.

Numerical relativity simulations have demonstrated that dynamical scalarization can significantly alter the late-time behavior of a BNS system. If this transition occurs before merger, the sudden growth of effective scalar couplings impacts the system's gravitational binding energy and energy flux so as to shorten the time to merger $[20,24,26]$.

The prospective detectability of this effect was investigated in Refs. [40,94] using Bayesian model selection.
The authors sought to recover injected inspiral waveforms containing dynamical scalarization with template banks constructed from similar waveforms. The injected signals and template banks used PN waveforms augmented with various nonanalytic models of dynamical scalarization. To mimic the abrupt activation of the dipole emission at the onset of dynamical scalarization, Ref. [94] added a -1 PN correction modulated by a Heaviside function to a GR waveform, i.e., signals of the form

$$
\tilde{h}(f)=\tilde{h}_{\mathrm{GR}}(f) e^{i \Psi_{-1 \mathrm{PN}}(f) \Theta\left(f-f_{*}\right)}
$$

where $\Psi_{-1 \mathrm{PN}}(f)=b f^{-7 / 3}$, and $b$ and $f_{*}$ are parameters of the model. Injected signals were recovered with a template bank of waveforms of the same form. In Ref. [40], the authors injected waveforms constructed in Ref. [25] by integrating the $2.5 \mathrm{PN}$ equations of motion combined with a semianalytic model of scalarization; they then performed parameter estimation using both templates, which included $-1 \mathrm{PN}$ and $0 \mathrm{PN}$ scalar-tensor effects throughout the entire inspiral and those that modeled their sudden activation as in Ref. [94].

Combined, these analyses provide a loose criterion for whether a dynamically scalarizing BNS system could be distinguished from the corresponding system in GR by aLIGO. The key characteristic of such systems is the frequency $f_{\mathrm{DS}}$ at which dynamical scalarization occurs. To be distinguishable from a GR waveform, a significant portion of the dynamically scalarized signal's SNR must occur after $f_{\mathrm{DS}}$, or equivalently, $f_{\mathrm{DS}}$ must be sufficiently lower than the merger frequency. Using waveforms of the form of Eq. (20), in Ref. [94] the authors found that dynamical scalarization can only be observed with aLIGO if $f_{\mathrm{DS}} \lesssim 50-100 \mathrm{~Hz}$. In only one injection considered in Ref. [40] was dynamical scalarization detectable, occurring at $f_{\mathrm{DS}} \approx 80 \mathrm{~Hz}$. Understandably, these analyses rely on some initial assumptions that may bias these estimates away from the real detectability criteria, such as the limited range of masses and EOS considered and ignoring any degeneracies introduced by the merger and ringdown portions of the waveform or by the inclusion of spins. Ignoring these subtleties for the moment, we investigate whether the pulsar-timing constraints described in Sec. III can exclude the possibility of observing dynamical scalarization with aLIGO using the conservative detectability criterion from Refs. [40,94] that scalarization must occur by $f_{\mathrm{DS}} \lesssim 50 \mathrm{~Hz}$.

We consider binary systems composed of NSs with masses ranging from $1.3 M_{\odot}$ to $1.9 M_{\odot}$. We compute $f_{\mathrm{DS}}$ within the "post-Dickean" (PD) framework, a resummation of the PN expansion formulated in Ref. [27]. This model introduces new dynamical d.o.f. that capture the nonperturbative growth of the scalar field using a semianalytic feedback loop. This approach provides a mathematically consistent backing to previous models of dynamical 
scalarization [25]. The model incorporates a certain flexibility in the choice of resummed quantities; we adopt the $\left(m^{(\mathrm{RE})}, F^{(\tilde{\varphi})}\right)$ scheme outlined in Table I of Ref. [27] because it was found to give the best agreement with numerical computations of quasiequilibrium configurations [26]. For clarity, we dress quantities defined in the PD framework with tildes and leave quantities defined in the PN framework unadorned; in the limit where no resummation is performed, the PD quantities reduce to their PN analogs.

Within the PD framework, the effective scalar coupling of each NS is promoted to a function of both the asymptotic scalar field $\varphi_{0}$ and the local scalar field in which the body is immersed, i.e., $\tilde{\alpha}_{A}=\tilde{\alpha}_{A}\left(\varphi_{0}, \varphi_{A}\right)$. Unlike in the PN treatment, this coupling evolves as the BNS coalesces. Similarly, the inertial mass of each body $\tilde{m}_{A}\left(\varphi_{0}, \varphi_{A}\right)$ evolves in the PD framework. However, this mass varies by no more than $0.01 \%$, so in practice, one can simply use the PN mass $m_{A}$ in place of $\tilde{m}_{A}$.

We define the mass-averaged scalar coupling of the system as

$$
\bar{\alpha} \equiv \frac{\tilde{m}_{A} \tilde{\alpha}_{A}+\tilde{m}_{B} \tilde{\alpha}_{B}}{\tilde{m}_{A}+\tilde{m}_{B}}
$$

where precise definitions of $\tilde{m}_{A}$ and $\tilde{\alpha}_{A}$ are given in Eqs. (A3) and (A4) in Ref. [27]. Note that for equal-mass binaries, we have $\tilde{\alpha}_{A}=\tilde{\alpha}_{B}=\bar{\alpha}$.

Following the work of Ref. [27], we compute the massaveraged scalar coupling as a function of frequency for binaries on quasicircular orbits to $1 \mathrm{PD}$ order. The average scalar coupling is plotted in Fig. 10 for equal-mass binaries for theories that saturate the pulsar-timing constraints at 90\% C.L.s, as given in Table II. Scalarization occurs earlier for larger mass systems, with an ordering (by EOS) determined by the magnitude of

$$
\beta_{A}=\left(\frac{\mathrm{d} \alpha_{A}}{\mathrm{~d} \varphi}\right)_{\varphi=\varphi_{0}} .
$$

To compute this quantity, one takes the difference in effective scalar couplings of NSs (of equal baryonic mass) with infinitesimally different asymptotic scalar fields $\varphi_{0}$;
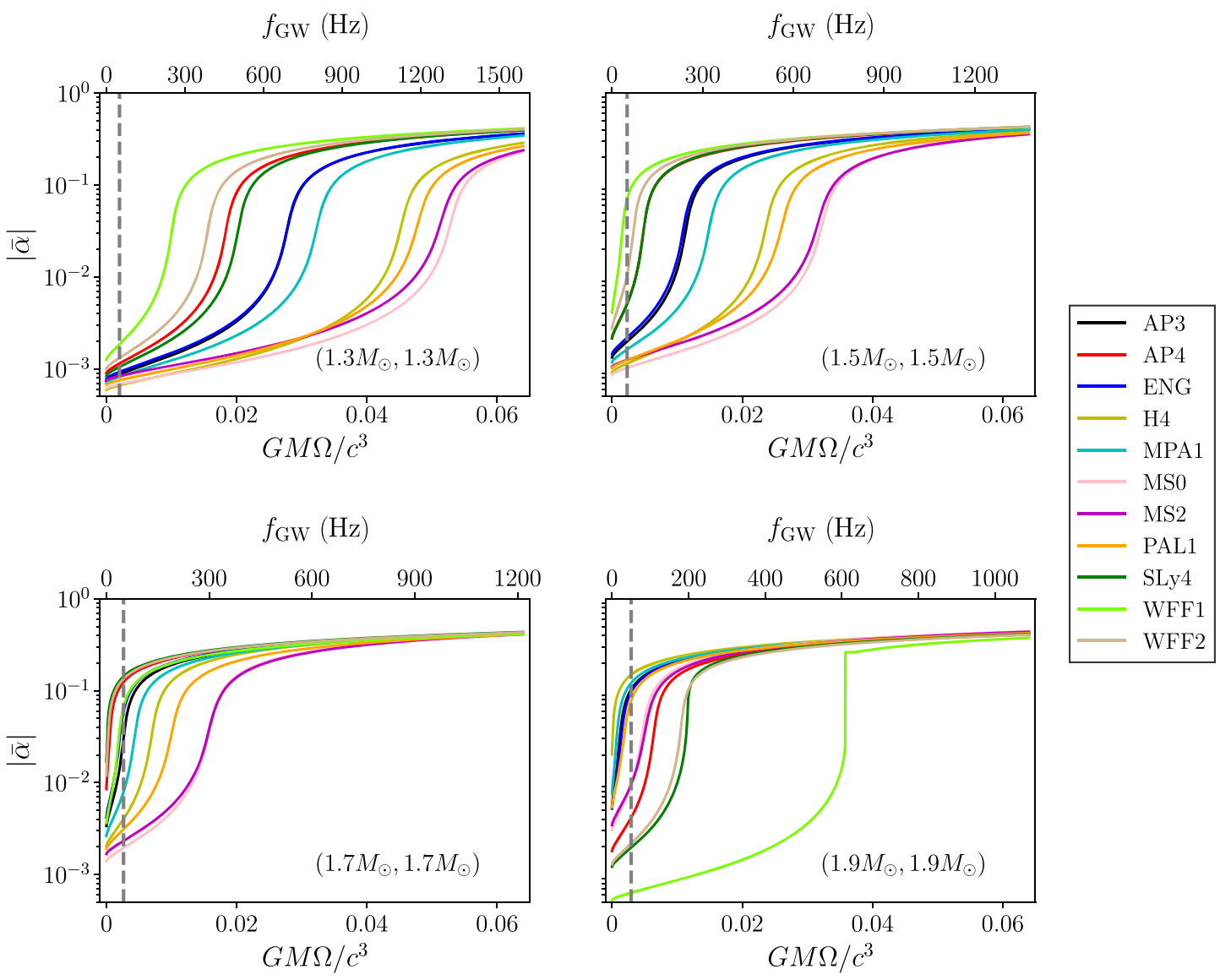

FIG. 10. Mass-averaged scalar coupling as a function of orbital angular frequency for equal-mass BNS systems with masses $\left(1.3 M_{\odot}, 1.3 M_{\odot}\right),\left(1.5 M_{\odot}, 1.5 M_{\odot}\right),\left(1.7 M_{\odot}, 1.7 M_{\odot}\right)$, and $\left(1.9 M_{\odot}, 1.9 M_{\odot}\right)$. We use the limits on $\left(\alpha_{0}, \beta_{0}\right)$ at $90 \%$ C.L.s, given in Table II, for each EOS. The corresponding GW frequency is given along the top axis, with $f_{\mathrm{GW}}=\Omega / \pi$. Dashed vertical lines highlight the conservative detectability criterion for aLIGO that $f_{\mathrm{DS}} \lesssim 50 \mathrm{~Hz}$, derived in Refs. [40,94]. 
TABLE IV. Frequency $f_{\mathrm{DS}}$ at which dynamical scalarization occurs for various equal-mass binaries, given in Hz. Results are given for theories that saturate the constraints given in Table II at $68 \%$ and $90 \%$ C.L.s. Binary systems are specified by their component NS masses, given in units of $M_{\odot}$. We highlight systems that scalarize at frequencies below $50 \mathrm{~Hz}$ with boldface.

\begin{tabular}{|c|c|c|c|c|c|c|c|c|}
\hline \multirow[b]{2}{*}{ EOS } & \multicolumn{4}{|c|}{$68 \%$ confidence level } & \multicolumn{4}{|c|}{$90 \%$ confidence level } \\
\hline & $1.3-1.3$ & $1.5-1.5$ & $1.7-1.7$ & $1.9-1.9$ & $1.3-1.3$ & $1.5-1.5$ & $1.7-1.7$ & $1.9-1.9$ \\
\hline AP3 & 838 & 354 & 123 & 84 & 694 & 246 & 50 & 20 \\
\hline AP4 & 577 & 183 & 57 & 199 & 461 & 105 & 8 & 109 \\
\hline ENG & 858 & 358 & 118 & 102 & 694 & 236 & 39 & 24 \\
\hline $\mathrm{H} 4$ & 1301 & 650 & 235 & 51 & 1131 & 513 & 131 & $<\mathbf{1}$ \\
\hline MPA1 & 955 & 436 & 162 & 67 & 809 & 325 & 84 & 11 \\
\hline MSO & 1503 & 854 & 422 & 165 & 1320 & 700 & 302 & 81 \\
\hline MS2 & 1471 & 843 & 426 & 177 & 1290 & 687 & 306 & 88 \\
\hline PAL1 & 1350 & 693 & 287 & 95 & 1190 & 570 & 193 & 32 \\
\hline SLy4 & 674 & 217 & 66 & 356 & 508 & 106 & $<1$ & 197 \\
\hline WFF1 & 386 & 118 & 128 & 841 & 251 & 33 & 35 & 608 \\
\hline WFF2 & 519 & 154 & 57 & 302 & 391 & 72 & $<1$ & 181 \\
\hline
\end{tabular}

however, for stars that are not spontaneously scalarized, $\beta_{A}$ is given approximately by

$$
\beta_{A} \approx \frac{\beta_{0}\left|\alpha_{A}\right|}{\left|\alpha_{0}\right|}
$$

provided that $\left|\alpha_{A}\right|$ is sufficiently small. Binaries with spontaneously scalarized stars begin with an appreciable effective scalar coupling at large separations that continues to grow as they coalesce. In light of this remark, we note that there is no observational distinction between spontaneous (or induced) scalarization and dynamical scalarization that occurs at sufficiently low frequencies; for example, compare the scalarization of $\left(1.7 M_{\odot}, 1.7 M_{\odot}\right)$ systems composed of NSs with the EOSs SLy4, AP4, and WFF1 (the dark green, red, and beige curves in the lower left panel of Fig. 10, respectively).

The sharp feature for the WFF1 EOS in the $\left(1.9 M_{\odot}\right.$, $\left.1.9 M_{\odot}\right)$ system occurs because of the relatively low mass at which spontaneous scalarization occurs for this particular EOS. We provide a more detailed analysis of this phenomenon in Appendix B. Similarly, abrupt transitions occur for other EOSs in more massive binary systems with individual masses $\gtrsim 2 M_{\odot}$.

We adopt the method introduced in Ref. [26] to extract $f_{\text {DS }}$. The average effective scalar coupling can be closely fit by the piecewise function

$$
\left(1+\bar{\alpha}^{2}\right)^{10 / 3}=1+a_{1}\left(x-x_{\mathrm{DS}}\right) \Theta\left(x-x_{\mathrm{DS}}\right),
$$

where $\Theta$ is the Heaviside function, $a_{1}$ and $x_{\mathrm{DS}}$ are fitting parameters, and $x \equiv\left(G_{*} M \Omega / c^{3}\right)^{2 / 3}$. In practice, we identify $x_{\mathrm{DS}}$ with the peak in the second derivative of the lefthand side of Eq. (24) with respect to $x$. The gravitational wave frequency at which dynamical scalarization occurs is then given by $f_{\mathrm{DS}}=\Omega_{\mathrm{DS}} / \pi$. In Ref. [27], the PD prediction was found to reproduce numerical-relativity results to within an error of $\lesssim 10 \%$ with this fitting procedure.

The dynamical scalarization frequencies for the configurations considered in Fig. 10 are given in Table IV for theories constrained at the $68 \%$ and $90 \%$ C.L.s. Systems containing spontaneously scalarized stars (i.e., those with

TABLE V. Frequency $f_{\text {DS }}$ (in Hz) at which dynamical scalarization occurs for various unequal-mass binaries with the EOS MPA1. Results are given for theories that saturate the constraints given in Table II at $68 \%$ and $90 \%$ C.L.s. Binary systems are specified by their

\begin{tabular}{|c|c|c|c|c|c|c|c|c|c|c|c|c|c|c|c|c|}
\hline & \multicolumn{8}{|c|}{$68 \%$ confidence level } & \multicolumn{8}{|c|}{$90 \%$ confidence level } \\
\hline & 1.2 & 1.3 & 1.4 & 1.5 & 1.6 & 1.7 & 1.8 & 1.9 & 1.2 & 1.3 & 1.4 & 1.5 & 1.6 & 1.7 & 1.8 & 1.9 \\
\hline 1.2 & 1340 & 1130 & 942 & 767 & 611 & 471 & 364 & 304 & 1160 & 973 & 788 & 618 & 458 & 315 & 194 & 119 \\
\hline 1.3 & $\ldots$ & 955 & 795 & 649 & 515 & 399 & 306 & 258 & $\ldots$ & 809 & 653 & 511 & 380 & 262 & 158 & 99 \\
\hline 1.4 & $\ldots$ & $\ldots$ & 661 & 538 & 427 & 329 & 253 & 212 & $\ldots$ & $\ldots$ & 529 & 415 & 305 & 210 & 129 & 79 \\
\hline 1.5 & $\ldots$ & $\ldots$ & $\ldots$ & 436 & 347 & 268 & 206 & 172 & $\ldots$ & $\ldots$ & $\ldots$ & 325 & 240 & 165 & 101 & 62 \\
\hline 1.6 & $\ldots$ & $\ldots$ & $\ldots$ & $\ldots$ & 274 & 212 & 163 & 136 & $\ldots$ & $\ldots$ & $\ldots$ & $\ldots$ & 178 & 120 & 73 & 46 \\
\hline 1.7 & $\ldots$ & $\ldots$ & $\ldots$ & $\ldots$ & $\ldots$ & 162 & 126 & 105 & $\ldots$ & $\ldots$ & $\ldots$ & $\ldots$ & $\ldots$ & 84 & 51 & 31 \\
\hline 1.8 & $\ldots$ & $\ldots$ & $\ldots$ & $\ldots$ & $\ldots$ & $\ldots$ & 96 & 80 & $\ldots$ & $\ldots$ & $\ldots$ & $\ldots$ & $\ldots$ & $\ldots$ & 32 & 19 \\
\hline 1.9 & $\ldots$ & $\ldots$ & $\ldots$ & $\ldots$ & $\ldots$ & $\ldots$ & $\ldots$ & 67 & $\ldots$ & $\ldots$ & $\ldots$ & $\ldots$ & $\ldots$ & $\ldots$ & $\ldots$ & 11 \\
\hline
\end{tabular}
component NS masses, given in units of $M_{\odot}$. We highlight systems that scalarize at frequencies below $50 \mathrm{~Hz}$ with boldface. 
appreciable effective scalar coupling even in isolation) are demarcated as scalarizing below $1 \mathrm{~Hz}$; as noted above, these systems would be indistinguishable to GW detectors from those that dynamically scalarize below $1 \mathrm{~Hz}$. For clarity, we highlight the systems in Table IV that scalarize (dynamically or spontaneously) below $50 \mathrm{~Hz}$. Recall that, under our definition, induced scalarization occurs in binaries comprised of one initially scalarized star and one initially unscalarized star; this asymmetry cannot be achieved in equal-mass systems like those discussed above.

We next consider the onset of dynamical scalarization in unequal-mass systems. For the sake of compactness, we show in Table $\mathrm{V}$ the dynamical scalarization frequencies for binaries with NS masses of $1.2 M_{\odot}$ to $1.9 M_{\odot}$ with just the MPA1 EOS. We find that the total mass plays a more important role in determining the onset of dynamical scalarization than the mass ratio. Fixing the total mass, we find that scalarization occurs earlier in more asymmetric binaries of lower mass (e.g., $M \lesssim 3.2 M_{\odot}$ for the MPA1 EOS). None of the systems listed in Table $\mathrm{V}$ undergoes induced scalarization. As before, we highlight the systems in Table $\mathrm{V}$ that scalarize below $50 \mathrm{~Hz}$.

To summarize, Tables IV and V demonstrate that binarypulsar constraints cannot entirely rule out the possibility of dynamical scalarization occurring at frequencies $f_{\mathrm{DS}} \lesssim$ $50 \mathrm{~Hz}$ at $90 \%$ C.L. Initial detectability studies—Refs. [40,94] discussed above - suggest that this early scalarization should be observable with aLIGO (although these conclusions should be confirmed with future studies in light of the limitations of these works; see above). Thus, failure to detect dynamical scalarization in future GW observations could provide tighter constraints on the parameters $\left(\alpha_{0}, \beta_{0}\right)$ in DEF theory than pulsar timing. However, as can be seen from Table IV, the prospects of producing such complementary constraints depend critically on the observed NS masses and the EOS of NSs.

\section{CONCLUSIONS}

In this paper, we have studied the scalarization phenomena $[14,24]$ in the massless mono-scalar-tensor theory of gravity of DEF with pulsar timing and laser-interferometer GW detectors on the Earth. We now summarize the key conclusions of our analysis.

(1) The spontaneous scalarization phenomenon [14] occurs at different NS mass ranges for different EOSs [20]. Therefore, in a well-timed relativistic binary-pulsar system with a specific NS mass, the scalar-tensor gravity might be stringently constrained for some EOSs whose spontaneousscalarization phenomenon occurs near that specific NS mass. However, in general, strong scalarization could still take place if NSs are described by an EOS whose scalarization occurs at a mass that is different from the one observed.
(2) Combining two well-timed binary-pulsar systems with quite different NS masses, one could, in principle, constrain the scalar-tensor theory with whatever EOS nature provides us. Using MCMC simulations, we showed in Sec. III that, by combining five binary pulsars $[35,38,49,53,54]$ that best constrain gravitational dipolar radiation, we can already bound the scalarization parameter $\beta_{0}$ to be $\gtrsim-4.28$ at $68 \%$ C.L. and $\gtrsim-4.38$ at $90 \%$ C.L., for any of the eleven EOSs that we have considered.

(3) Nevertheless, because of the limited distribution of masses of the five chosen binary pulsars, we found that if the EOSs of NSs were similar to the ones of AP4, SLy4, or WFF2, NSs with masses of $m_{A} \simeq$ 1.70-1.73 $M_{\odot}$ could still develop an effective scalar coupling $\gtrsim \mathcal{O}\left(10^{-2}\right)$. This is also true for the EOS H4 with $m_{A} \simeq 1.92 M_{\odot}$, and for the EOSs MSO and MS2 with $m_{A} \simeq 2.26 M_{\odot}$ (see Fig. 5).

(4) Using the upper limits on the effective scalar coupling of NSs from binary pulsars, we found that for BNSs in the frequency bands of aLIGO, CE, and ET, we could still have a large time-domain dephasing in the number of $\mathrm{GW}$ cycles, on the orders of $\mathcal{O}\left(10^{1}\right), \mathcal{O}\left(10^{2}\right)$, and $\mathcal{O}\left(10^{3}\right)$, respectively (see Table III).

(5) We performed a Fisher-matrix study of BNS inspiral signals using aLIGO, CE, and ET. We found that for BNSs at a luminosity distance $D_{L}=200 \mathrm{Mpc}$, where we expect to observe those sources, aLIGO can still improve the limits from binary pulsars for a couple of EOSs with BNSs of suitable masses. CE (whose bandwidth starts at $5 \mathrm{~Hz}$ ) can improve the current limits for all EOSs, while ET (whose bandwidth starts at $1 \mathrm{~Hz}$ ) will provide us with even more significant improvements over current constraints for all EOSs. This is mainly because of better low-frequency sensitivity. Our conclusions for aLIGO differ from the one obtained in Refs. [16,36,37], where the authors concluded that pulsar timing would do better than aLIGO in constraining scalar-tensor theories. The main reason for this difference comes from a better understanding and larger span of the NS masses and EOSs during the past two decades [34,35], and the different PSD for aLIGO used in Refs. [16,71]. If we restricted the analysis to the same NS masses and the same EOS used in Ref. [16], we would recover the same conclusions as in Ref. [16] (see Fig. 7).

(6) We investigated dynamical scalarization in equal-mass and unequal-mass BNS systems. With the criterion that the dynamical scalarization transition frequency must fall below about $50 \mathrm{~Hz}[40,94]$ to be detectable, we found that aLIGO could observe this phenomenon given the constraints obtained from binary-pulsar timing, even away from the scalarization windows. 
We found that the prospects for observing dynamical scalarization with GW detectors depends critically on the NS EOS-for example, dynamical scalarization of NSs with the MSO EOS could not be detected with aLIGO. Producing new constraints on scalar-tensor theories from GW searches for dynamical scalarization requires waveform models that can faithfully reproduce this nonperturbative phenomenon; ultimately, these conclusions should be revisited once such models are developed.

Our comparisons between binary pulsars and GWs made use of the current limits of the former and the expected limits of the latter. We show that advanced and nextgeneration ground-based GW detectors have the potential to further improve the current limits set by pulsar timing. Nevertheless, the binary-pulsar limits will also improve over time, especially if suitable systems filling the scalarization windows are discovered in future pulsar surveys. Better mass measurements of currently known pulsars will also help in narrowing down the constraints, especially with PSRs J1012 + 5307 [53] and J1913 + 1102 [87], whose observational uncertainties in masses are still large, and they might have the right masses to close the windows below $2 M_{\odot}$. To reach this goal, the next generation of radio telescopes, such as FAST and SKA, will play a particularly important role $[22,95]$.

\section{ACKNOWLEDGMENTS}

We thank Paulo Freire, Ian Harry, Jan Steinhoff, Thomas Tauris, and Nicolás Yunes for helpful discussions. We are grateful to Jim Lattimer for providing us with tabulated data for neutron-star equations of state. The Markov-chain Monte Carlo runs were performed on the VULCAN cluster at the Max Planck Institute for Gravitational Physics in Potsdam.

\section{APPENDIX A: INGREDIENTS FOR THE FISHER-MATRIX ANALYSIS}

For a GW detector with the one-side PSD, $S_{n}(f)$, the SNR of a Fourier-domain waveform, $\tilde{h}(f)$, is

$$
\rho=(\tilde{h}(f) \mid \tilde{h}(f))^{1 / 2},
$$

where the inner product is defined to be $[82,83]$

$\left(\tilde{h}_{1}(f) \mid \tilde{h}_{2}(f)\right) \equiv 2 \int_{f_{\min }}^{f_{\max }} \frac{\tilde{h}_{1}^{*}(f) \tilde{h}_{2}(f)+\tilde{h}_{1}(f) \tilde{h}_{2}^{*}(f)}{S_{n}(f)} \mathrm{d} f$.

For all calculations in Sec. IV A, we use the design zerodetuned high-power noise PSD, starting from $10 \mathrm{~Hz}$ for aLIGO [75], the target noise PSD, starting from $5 \mathrm{~Hz}$ for CE [23], and the ET-D noise PSD, starting from $1 \mathrm{~Hz}$ for ET [74]. Thus, in Eq. (A2), we choose $f_{\min }=10 \mathrm{~Hz}, 5 \mathrm{~Hz}$, and $1 \mathrm{~Hz}$ for aLIGO, CE, and ET, respectively. Somewhat arbitrarily, we choose for $f_{\max }$ twice the innermost stable circular orbit (ISCO) frequency computed from the binary's binding energy at 2 PN order. It reads [96]

$$
f_{\max }=\frac{c^{3}}{\pi G M}\left[\frac{3}{14 \eta}\left(1-\sqrt{1-\frac{14}{9} \eta}\right)\right]^{3 / 2} .
$$

Because the three detectors do not have good sensitivity at high frequency, say $\gtrsim \mathrm{kHz}$, the choice of $f_{\text {max }}$ influences the result very marginally.

For the nonspinning BNS inspiraling waveform in the Fourier domain, we use a restricted waveform with the leading-order term in amplitude $\mathcal{A}$ and up to $3.5 \mathrm{PN}$ terms in the phase $\Psi(f)[17,71,76]$,

$$
\tilde{h}(f)=\mathcal{A} f^{-7 / 6} e^{i \Psi(f)},
$$

where $\mathfrak{t} \equiv \pi G M f / c^{3} ; \mathcal{A} \propto \mathcal{M}^{5 / 6} / D_{L}$ with the chirp mass $\mathcal{M} \equiv \eta^{3 / 5} M$ and the luminosity distance $D_{L} ; t_{c}$ and $\Phi_{c}$ are reference time and phase, respectively; and $\gamma_{\mathrm{E}}=$ $0.577216 \ldots$ is the Euler constant. Note that in Eq. (A5), the gothic $\mathfrak{i t}$ is equal to $\eta^{-3 / 5} u$, where $u \equiv \pi G \mathcal{M} f / c^{3}$, as defined in Ref. [71]. In Eq. (A5), we include only the leading dipole term for the scalar contribution. Furthermore, since the spins of BNS systems are supposed to be 
small, we do not include them in the analysis (see Table III, where we give a rough estimation of the spin terms in the GW phasing).

To calculate the Fisher matrix (17), we need to compute partial derivatives of the frequency-domain waveform (A4). They read (notice that, when calculating derivatives, $\mathfrak{t}$ depends on both $\eta$ and $\mathcal{M}$ )

$$
\begin{aligned}
& \frac{\partial \tilde{h}(f)}{\partial \ln \mathcal{A}}=\tilde{h}(f), \\
& \frac{\partial \tilde{h}(f)}{\partial \ln \eta}= \frac{i}{\eta} \mathfrak{t}^{-5 / 3}\left\{-\frac{1}{3584} \Delta \alpha^{2} \mathfrak{t}^{-2 / 3}+\left(-\frac{743}{16128}+\frac{11}{128} \eta\right) \mathfrak{t}^{2 / 3}+\frac{9}{40} \pi \mathfrak{t}+\left(-\frac{3058673}{5419008}+\frac{5429}{21504} \eta+\frac{617}{512} \eta^{2}\right) \mathfrak{t}^{4 / 3}\right. \\
&+\pi\left(-\frac{7729}{4032}-\frac{38645}{32256} \ln \mathfrak{t}+\frac{13}{128} \eta\right) \mathfrak{t}^{5 / 3}+\left[-\frac{11328104339891}{166905446400}+6 \pi^{2}+\frac{321}{35} \gamma_{\mathrm{E}}+\frac{107}{35} \ln (64 \mathfrak{u})\right. \\
&\left.\left.+\left(\frac{3147553127}{130056192}-\frac{451}{512} \pi^{2}\right) \eta+\frac{15211}{18432} \eta^{2}-\frac{25565}{6144} \eta^{3}\right] \mathfrak{t}^{2}+\pi\left(-\frac{15419335}{1548288}-\frac{75703}{32256} \eta-\frac{14809}{10752} \eta^{2}\right) \mathfrak{t}^{7 / 3}\right\} \tilde{h}(f), \\
& \frac{\partial \tilde{h}(f)}{\partial \ln \mathcal{M}}= \frac{i}{\eta} \mathfrak{u}^{-5 / 3}\left\{-\frac{5}{128}+\frac{5}{3072}(\Delta \alpha)^{2} \mathfrak{u}^{-2 / 3}+\left(-\frac{3715}{32256}-\frac{55}{384} \eta\right) \mathfrak{t}^{2 / 3}+\frac{\pi}{4} \mathfrak{t}+\left(-\frac{15293365}{65028096}-\frac{27145}{64512} \eta-\frac{3085}{9216} \eta^{2}\right) \mathfrak{t}^{4 / 3}\right. \\
&+\pi\left(\frac{38645}{32256}-\frac{65}{384} \eta\right) \mathfrak{t}^{5 / 3}+\left[\frac{10052469856691}{600859607040}-\frac{5}{3} \pi^{2}-\frac{107}{42} \gamma_{\mathrm{E}}-\frac{107}{126} \ln (64 \mathfrak{u t})\right. \\
&+\left.\left(-\frac{15737765635}{390168576}+\frac{2255}{1536} \pi^{2}\right) \eta+\frac{76055}{221184} \eta^{2}-\frac{127825}{165888} \eta^{3}\right] \mathfrak{t}^{2} \\
&+\left.\pi\left(\frac{77096675}{16257024}+\frac{378515}{96768} \eta-\frac{74045}{48384} \eta^{2}\right) \mathfrak{u}^{7 / 3}\right\} \tilde{h}(f),
\end{aligned}
$$$$
\frac{\partial \tilde{h}(f)}{\partial t_{c}}=i 2 \pi f \tilde{h}(f)
$$$$
\frac{\partial \tilde{h}(f)}{\partial \Phi_{c}}=-i \tilde{h}(f)
$$$$
\frac{\partial \tilde{h}(f)}{\partial(\Delta \alpha)^{2}}=-i \frac{5}{7168 \eta} \mathfrak{t}^{-7 / 3} \tilde{h}(f) .
$$

\section{APPENDIX B: DYNAMICAL SCALARIZATION IN ULTRARELATIVISTIC BINARY NEUTRON STARS}

In this appendix, we discuss the sharp feature observed in the averaged effective scalar coupling of a very massive BNS that undergoes dynamical scalarization (see the $1.9 M_{\odot}-1.9 M_{\odot}$ case in Fig. 10). We find that, generically, NSs of very high mass can scalarize more abruptly than their less massive counterparts.

From Fig. 5, we observe that very massive NSs exhibit very small effective scalar couplings $\alpha_{A}$. In these stars, the effective scalar coupling is nonperturbatively suppressed below the nonrelativistic (low-mass) limit $\alpha_{A} \approx \alpha_{0}$. The cores of these stars are ultrarelativistic, with a negative trace of the stress-energy tensor $T_{*}=\epsilon_{*}-3 p_{*}<0$.

The mass at which NSs become ultrarelativistic, in this sense, depends on the EOS and can be read off from Fig. 7 as the mass at which the best constraint on $|\alpha|$ drops to zero. Recall that spontaneous scalarization stems from a large, positive source on the right-hand side of Eq. (3) that grows with $\varphi$. In ultrarelativistic stars, this source term becomes negative, causing the star to spontaneously "descalarize."

When placed in a binary system, ultrarelativistic NSs can dynamically scalarize, but the transition occurs very abruptly (e.g., the $1.9 M_{\odot}-1.9 M_{\odot}$ system with the EOS WFF1 shown in Fig. 10). As the system scalarizes, the massive NSs transition to a state in which $T_{*}$ is everywhere positive. Figure 11 depicts this transition in comparison to dynamical scalarization in less massive systems. The top panel shows $\tilde{\beta}_{A}$-the PD equivalent of the quantity defined in Eq. (22)—for $1.8 M_{\odot}$ (solid line) and $1.9 M_{\odot}$ (dashed line) stars with the EOS WFF1 plotted as a function of the scalar field. The highlighted points indicate the field at each NS in an equal-mass binary before (blue), during (red), and after (green) dynamical scalarization. The bottom panels depict the profile of $T_{*}$ within each star at each of these points.

The top panel of Fig. 11 demonstrates why dynamical scalarization occurs abruptly for ultrarelativistic NSs. 

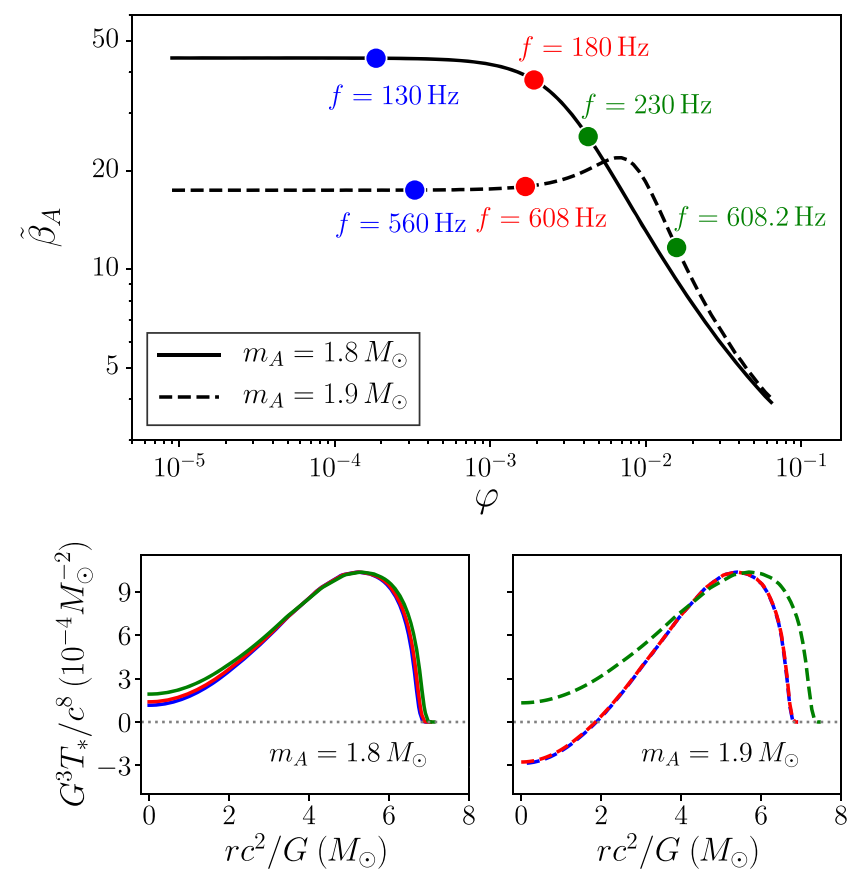

FIG. 11. (Top panel) Evolution of $\tilde{\beta}_{A}$ for NSs with (dashed line) and without (solid line) ultrarelativistic cores as a function of the background scalar field. The blue, red, and green annotations indicate the scalar field at each star at frequencies before, during, and after dynamical scalarization in an equal-mass binary system, respectively. (Bottom panels) Profile of the trace of the stressenergy tensor within each NS at each of the annotated points in the top panel.

Recall that $\beta_{A}$ (and consequently $\tilde{\beta}_{A}$ ) quantifies how easily a NS can scalarize, with larger values indicating that the star is more susceptible to dynamical scalarization. As expected, when immersed in a weak scalar field (i.e., during the early inspiral), $\tilde{\beta}_{A}$ is significantly smaller for ultrarelativistic stars than less massive stars, indicating that the latter will dynamically scalarize at a lower frequency. However, unlike for less massive stars, $\tilde{\beta}_{A}$ increases slightly as the scalar field reaches larger values $(\varphi \sim 0.002$ in Fig. 11) for ultrarelativistic NSs. This triggers a runaway process in a binary system, as a small increase in the scalar field produced by one star causes the other star to scalarize more easily, which in turn allows the second star to produce a larger scalar field for the first. For the ultrarelativistic BNS depicted in the top panel of Fig. 11, this transition is completed after an evolution of only $0.2 \mathrm{~Hz}$.

[1] T. Kaluza, Zum Unitätsproblem der Physik, Sitzungsberichte der Königlich Preußischen Akademie der Wissenschaften (Berlin), 966 (1921).

[2] T. Clifton, P. G. Ferreira, A. Padilla, and C. Skordis, Modified Gravity and Cosmology, Phys. Rep. 513, 1 (2012).
[3] P. Jordan, Formation of the Stars and Development of the Universe, Nature (London) 164, 637 (1949).

[4] P. Jordan, Schwerkraft und Weltall, Die Wissenschaft, Bd. 107 (Braunschweig, 1952).

[5] M. Fierz, On the Physical Interpretation of P. Jordan's Extended Theory of Gravitation, Helv. Phys. Acta 29, 128 (1956).

[6] C. Brans and R. H. Dicke, Mach's Principle and a Relativistic Theory of Gravitation, Phys. Rev. 124, 925 (1961).

[7] T. Damour and G. Esposito-Farèse, Tensor Multiscalar Theories of Gravitation, Classical Quantum Gravity 9, 2093 (1992).

[8] C. M. Will, Theory and Experiment in Gravitational Physics (Cambridge University Press, Cambridge, 1993).

[9] G. W. Horndeski, Second-Order Scalar-Tensor Field Equations in a Four-Dimensional Space, Int. J. Theor. Phys. 10, 363 (1974).

[10] C. M. Will, The Confrontation between General Relativity and Experiment, Living Rev. Relativity 17, 4 (2014).

[11] E. Berti et al., Testing General Relativity with Present and Future Astrophysical Observations, Classical Quantum Gravity 32, 243001 (2015).

[12] L. Shao and N. Wex, Tests of Gravitational Symmetries with Radio Pulsars, Sci. China Phys. Mech. Astron. 59, 699501 (2016).

[13] L. Shao, Testing the Strong Equivalence Principle with the Triple Pulsar PSR J0337 + 1715, Phys. Rev. D 93, 084023 (2016).

[14] T. Damour and G. Esposito-Farèse, Nonperturbative Strong Field Effects in Tensor-Scalar Theories of Gravitation, Phys. Rev. Lett. 70, 2220 (1993).

[15] T. Damour and G. Esposito-Farèse, Tensor-Scalar Gravity and Binary Pulsar Experiments, Phys. Rev. D 54, 1474 (1996).

[16] T. Damour and G. Esposito-Farèse, Gravitational Wave versus Binary-Pulsar Tests of Strong Field Gravity, Phys. Rev. D 58, 042001 (1998).

[17] E. Berti, A. Buonanno, and C. M. Will, Estimating Spinning Binary Parameters and Testing Alternative Theories of Gravity with LISA, Phys. Rev. D 71, 084025 (2005).

[18] E. Berti, A. Buonanno, and C. M. Will, Testing General Relativity and Probing the Merger History of Massive Black Holes with LISA, Classical Quantum Gravity 22, S943 (2005).

[19] K. Yagi and T. Tanaka, DECIGO/BBO as a Probe to Constrain Alternative Theories of Gravity, Prog. Theor. Phys. 123, 1069 (2010).

[20] M. Shibata, K. Taniguchi, H. Okawa, and A. Buonanno, Coalescence of Binary Neutron Stars in a Scalar-Tensor Theory of Gravity, Phys. Rev. D 89, 084005 (2014).

[21] N. Yunes, K. Yagi, and F. Pretorius, Theoretical Physics Implications of the Binary Black-Hole Mergers GW150914 and GW151226, Phys. Rev. D 94, 084002 (2016).

[22] L. Shao et al., Testing Gravity with Pulsars in the SKA Era, Proc. Sci. (AASKA14) 042 [arXiv:1501.00058].

[23] B. P. Abbott et al. (LIGO Scientific), Exploring the Sensitivity of Next Generation Gravitational Wave Detectors, Classical Quantum Gravity 34, 044001 (2017). 
[24] E. Barausse, C. Palenzuela, M. Ponce, and L. Lehner, Neutron-Star Mergers in Scalar-Tensor Theories of Gravity, Phys. Rev. D 87, 081506 (2013).

[25] C. Palenzuela, E. Barausse, M. Ponce, and L. Lehner, Dynamical Scalarization of Neutron Stars in Scalar-Tensor Gravity Theories, Phys. Rev. D 89, 044024 (2014).

[26] K. Taniguchi, M. Shibata, and A. Buonanno, Quasiequilibrium Sequences of Binary Neutron Stars Undergoing Dynamical Scalarization, Phys. Rev. D 91, 024033 (2015).

[27] N. Sennett and A. Buonanno, Modeling Dynamical Scalarization with a Resummed Post-Newtonian Expansion, Phys. Rev. D 93, 124004 (2016).

[28] J. Aasi et al. (LIGO Scientific), Advanced LIGO, Classical Quantum Gravity 32, 074001 (2015).

[29] T. Damour, Physics of Relativistic Objects in Compact Binaries: From Birth to Coalescence, Astrophysics and Space Science Library, Vol. 359 (Springer, Netherlands, 2009), p. 1.

[30] For black holes, the effective scalar coupling equals zero. Therefore, the tests performed with binary black holes [31] do not directly apply to the DEF theory.

[31] B. P. Abbott et al. (Virgo and LIGO Scientific), Tests of General Relativity with GW150914, Phys. Rev. Lett. 116, 221101 (2016).

[32] For sufficiently negative $\beta_{0}(\lesssim-4.6)$, NSs do not descalarize before reaching their maximum mass; i.e. spontaneous scalarization is found for all NSs above a certain critical mass, which depends on the actual value of $\beta_{0}$ and the EOS $[14,15]$.

[33] In this paper, generally we denote with $n \mathrm{PN}$ the $\mathcal{O}\left(v^{2 n} / c^{2 n}\right)$ corrections to the leading Newtonian dynamics (equations of motion). Therefore, the gravitational dipolar radiation reaction is at $1.5 \mathrm{PN}$, and the quadrupolar radiation is at $2.5 \mathrm{PN}$. In the GW phasing, when there is no potential confusion, we sometimes refer to the quadrupolar (dipolar) radiation as $0 \mathrm{PN}$ $(-1 \mathrm{PN})$, as typically done in the literature.

[34] P. Demorest, T. Pennucci, S. Ransom, M. Roberts, and J. Hessels, Shapiro Delay Measurement of a Two Solar Mass Neutron Star, Nature (London) 467, 1081 (2010).

[35] J. Antoniadis et al., A Massive Pulsar in a Compact Relativistic Binary, Science 340, 1233232 (2013).

[36] G. Esposito-Farèse, Binary Pulsar Tests of Strong Field Gravity and Gravitational Radiation Damping, in Proceedings of the Tenth Marcel Grossmann Meeting. On Recent Developments in Theoretical and Experimental General Relativity, Gravitation and Relativistic Field Theories (World Scientific Publishing, Singapore, 2004), pp. 647-666.

[37] G. Esposito-Farèse, Tests of Scalar-Tensor Gravity, Phi in the Sky: The Quest for Cosmological Scalar Fields, AIP Conf. Proc. 736, 35 (2004).

[38] P. C. C. Freire, N. Wex, G. Esposito-Farèse, J. P. W. Verbiest, M. Bailes, B. A. Jacoby, M. Kramer, I. H. Stairs, J. Antoniadis, and G. H. Janssen, The Relativistic PulsarWhite Dwarf Binary PSR J1738+0333 II. The Most Stringent Test of Scalar-Tensor Gravity, Mon. Not. R. Astron. Soc. 423, 3328 (2012).

[39] N. Wex, Testing Relativistic Gravity with Radio Pulsars, arXiv:1402.5594.

[40] L. Sampson, N. Yunes, N. Cornish, M. Ponce, E. Barausse, A. Klein, C. Palenzuela, and L. Lehner, Projected
Constraints on Scalarization with Gravitational Waves from Neutron Star Binaries, Phys. Rev. D 90, 124091 (2014).

[41] T. Damour and K. Nordtvedt, General Relativity as a Cosmological Attractor of Tensor Scalar Theories, Phys. Rev. Lett. 70, 2217 (1993).

[42] T. Damour and K. Nordtvedt, Tensor-Scalar Cosmological Models and Their Relaxation Toward General Relativity, Phys. Rev. D 48, 3436 (1993).

[43] D. Anderson, N. Yunes, and E. Barausse, The Effect of Cosmological Evolution on Solar System Constraints and on the Scalarization of Neutron Stars in Massless ScalarTensor Theories, Phys. Rev. D 94, 104064 (2016).

[44] For cosmologies in the scalar-tensor theories with a positive $\beta_{0}$, we refer readers to Refs. $[41,42,45]$.

[45] D. Anderson and N. Yunes, Solar System Constraints on Massless Scalar-Tensor Gravity with Positive Coupling Constant upon Cosmological Evolution of the Scalar Field, Phys. Rev. D 96, 064037 (2017).

[46] F. M. Ramazanoğlu and F. Pretorius, Spontaneous Scalarization with Massive Fields, Phys. Rev. D 93, 064005 (2016).

[47] S. S. Yazadjiev, D. D. Doneva, and D. Popchev, Slowly Rotating Neutron Stars in Scalar-Tensor Theories with a Massive Scalar Field, Phys. Rev. D 93, 084038 (2016).

[48] T. A. de Pirey Saint Alby and N. Yunes, Cosmological Evolution and Solar System Consistency of Massive ScalarTensor Gravity, Phys. Rev. D 96, 064040 (2017).

[49] I. Cognard et al., A Massive-Born Neutron Star with a Massive White Dwarf Companion, Astrophys. J. 844, 128 (2017).

[50] An exception is Ref. [51], where, for individual binary pulsars, the most conservative limits in the $\left(\alpha_{0}, \beta_{0}\right)$ parameter space across different EOSs are presented.

[51] M. Kramer, Pulsars as Probes of Gravity and Fundamental Physics, Int. J. Mod. Phys. D 25, 1630029 (2016).

[52] W. Del Pozzo and A. Vecchio, On Tests of General Relativity with Binary Radio Pulsars, Mon. Not. R. Astron. Soc. 462, L21 (2016).

[53] K. Lazaridis et al., Generic Tests of the Existence of the Gravitational Dipole Radiation and the Variation of the Gravitational Constant, Mon. Not. R. Astron. Soc. 400, 805 (2009).

[54] D. J. Reardon et al., Timing Analysis for 20 Millisecond Pulsars in the Parkes Pulsar Timing Array, Mon. Not. R. Astron. Soc. 455, 1751 (2016).

[55] J. M. Lattimer, The Nuclear Equation of State and Neutron Star Masses, Annu. Rev. Nucl. Part. Sci. 62, 485 (2012).

[56] J. M. Lattimer and M. Prakash, Neutron Star Structure and the Equation of State, Astrophys. J. 550, 426 (2001).

[57] P. J. McMillan, The Mass Distribution and Gravitational Potential of the Milky Way, Mon. Not. R. Astron. Soc. 465, 76 (2017).

[58] P. J. Callanan, P. M. Garnavich, and D. Koester, The Mass of the Neutron Star in the Binary Millisecond Pulsar PSR J1012 + 5307, Mon. Not. R. Astron. Soc. 298, 207 (1998).

[59] J. Antoniadis, T. M. Tauris, F. Ozel, E. Barr, D. J. Champion, and P. C. C. Freire, The Millisecond Pulsar Mass Distribution: Evidence for Bimodality and Constraints on the Maximum Neutron Star Mass, arXiv:1605.01665. 
[60] J. Antoniadis, M. H. van Kerkwijk, D. Koester, P. C. C. Freire, N. Wex, T. M. Tauris, M. Kramer, and C. G. Bassa, The Relativistic Pulsar-White Dwarf Binary PSR J1738+ 0333 I. Mass Determination and Evolutionary History, Mon. Not. R. Astron. Soc. 423, 3316 (2012).

[61] Actually, in the full calculation, we need some other quantities as well-for example, the orbital period $P_{b}$ and the orbital eccentricity $e$. Those quantities are observationally very well determined (see Table I); thus, we use their central values and find that our constraints on $\left(\alpha_{0}, \beta_{0}\right)$ do not change on a relevant scale when we take into account the errors on those quantities.

[62] B. Bertotti, L. Iess, and P. Tortora, A Test of General Relativity Using Radio Links with the Cassini Spacecraft, Nature (London) 425, 374 (2003).

[63] J. Goodman and J. Weare, Ensemble Samplers with Affine Invariance, Commun. Appl. Math. Comput. Sci. 5, 65 (2010).

[64] D. Foreman-Mackey, D. W. Hogg, D. Lang, and J. Goodman, emcee: The MCMC Hammer, Publ. Astron. Soc. Pac. 125, 306 (2013).

[65] See http://dan.iel.fm/emcee.

[66] The masses in PSRs J0348 + 0432, J1012 + 5307, and $\mathrm{J} 1738+0333$ are based on a combination of radio timing of the pulsars and optical spectroscopic observation of the WDs. The derivation of the masses only depends on the well-understood WD atmosphere model in combination with gravity at Newtonian order, and the mass ratio $q$, which is free of any explicit strong-field effects [29]. Therefore, even within the DEF theory, these masses are valid [35,38]. For PSRs J1909 - 3744 and J2222 - 0137, the masses are derived from the range and shape of the Shapiro delay [15,39]. Since for the weakly self-gravitating WD companion $\left|\alpha_{B}\right| \approx\left|\alpha_{0}\right| \ll 1$, these masses are practically identical to the GR masses in Table I.

[67] P. C. Peters, Gravitational Radiation and the Motion of Two Point Masses, Phys. Rev. 136, B1224 (1964).

[68] In this context, see footnote "d" in Ref. [36], concerning WDs and very large (positive) $\beta_{0}$.

[69] A. Gelman and D. B. Rubin, Inference from Iterative Simulation Using Multiple Sequences, Stat. Sci. 7, 457 (1992).

[70] S. Brooks, A. Gelman, G. Jones, and X. L. Meng, Handbook of Markov Chain Monte Carlo (CRC Press, London, 2011).

[71] C. M. Will, Testing Scalar-Tensor Gravity with Gravitational Wave Observations of Inspiraling Compact Binaries, Phys. Rev. D 50, 6058 (1994).

[72] Michele Maggiore, Gravitational Waves. Vol. I: Theory and Experiments, Oxford Master Series in Physics (Oxford University Press, New York, 2007).

[73] N. Sennett, S. Marsat, and A. Buonanno, Gravitational Waveforms in Scalar-Tensor Gravity at 2PN Relative Order, Phys. Rev. D 94, 084003 (2016).

[74] S. Hild et al., Sensitivity Studies for Third-Generation Gravitational Wave Observatories, Classical Quantum Gravity 28, 094013 (2011).

[75] David Shoemaker, Advanced LIGO Anticipated Sensitivity Curves, LIGO Document No. T0900288-v3.

[76] A. Buonanno, B. Iyer, E. Ochsner, Y. Pan, and B. S. Sathyaprakash, Comparison of Post-Newtonian Templates for Compact Binary Inspiral Signals in Gravitational-Wave Detectors, Phys. Rev. D 80, 084043 (2009).

[77] M. Kramer et al., Tests of General Relativity from Timing the Double Pulsar, Science 314, 97 (2006).

[78] J. W. T. Hessels, S. M. Ransom, I. H. Stairs, P. C. Carvalho Freire, V. M. Kaspi, and F. Camilo, A Radio Pulsar Spinning at 716-Hz, Science 311, 1901 (2006).

[79] Notice that PSR J1748 - 2446ad is not in a double NS binary. Its companion is probably a bloated main-sequence star that recycles the pulsar to a large spin [78].

[80] W. Del Pozzo, K. Grover, I. Mandel, and A. Vecchio, Testing General Relativity with Compact Coalescing Binaries: Comparing Exact and Predictive Methods to Compute the Bayes Factor, Classical Quantum Gravity 31, 205006 (2014).

[81] J. Veitch et al., Parameter Estimation for Compact Binaries with Ground-Based Gravitational-Wave Observations Using the LALInference Software Library, Phys. Rev. D 91, 042003 (2015).

[82] L.S. Finn, Detection, Measurement and Gravitational Radiation, Phys. Rev. D 46, 5236 (1992).

[83] C. Cutler and E. E. Flanagan, Gravitational Waves from Merging Compact Binaries: How Accurately Can One Extract the Binary's Parameters from the Inspiral Wave Form?, Phys. Rev. D 49, 2658 (1994).

[84] M. Vallisneri, Use and Abuse of the Fisher Information Matrix in the Assessment of Gravitational-Wave ParameterEstimation Prospects, Phys. Rev. D 77, 042001 (2008).

[85] B. P. Abbott et al., Prospects for Observing and Localizing Gravitational-Wave Transients with Advanced LIGO and Advanced Virgo, Living Rev. Relativity 19, 1 (2016).

[86] P. A. R. Ade et al. (Planck Collaboration), Planck 2015 Results. XIII. Cosmological Parameters, Astron. Astrophys. 594, A13 (2016).

[87] P. Lazarus et al., Einstein@Home Discovery of a DoubleNeutron Star Binary in the PALFA Survey, Astrophys. J. 831, 150 (2016).

[88] In principle, there is still a weak dependence on $\alpha_{0}$ in $\mathcal{A}_{\beta_{0}}^{(A)}$. However, this dependence becomes very small for $\left|\alpha_{0}\right| \lesssim 10^{-2}$, as it scales with $\alpha_{0}^{2}$. Therefore, the $\alpha_{0}$ dependence is absolutely negligible for the parameter space explored here.

[89] K. G. Arun and A. Pai, Tests of General Relativity and Alternative Theories of Gravity Using Gravitational Wave Observations, Int. J. Mod. Phys. D 22, 1341012 (2013).

[90] X. Zhang, J. Yu, T. Liu, W. Zhao, and A. Wang, Testing Brans-Dicke Gravity Using the Einstein Telescope, Phys. Rev. D 95, 124008 (2017).

[91] F. Mignard and S. A. Klioner, Gaia: Relativistic Modelling and Testing, in Relativity in Fundamental Astronomy: Dynamics, Reference Frames, and Data Analysis, IAU Symposium, Vol. 261, edited by S. A. Klioner, P. K. Seidelmann, and M. H. Soffel (Cambridge University Press, Cambridge, 2010), pp. 306-314.

[92] R. F. P. Mendes, Possibility of Setting a New Constraint to Scalar-Tensor Theories, Phys. Rev. D 91, 064024 (2015).

[93] C. Palenzuela and S. L. Liebling, Constraining ScalarTensor Theories of Gravity from the Most Massive Neutron Stars, Phys. Rev. D 93, 044009 (2016). 
[94] L. Sampson, N. Cornish, and N. Yunes, Mismodeling in Gravitational-Wave Astronomy: The Trouble with Templates, Phys. Rev. D 89, 064037 (2014).

[95] R. Nan, D. Li, C. Jin, Q. Wang, L. Zhu, W. Zhu, H. Zhang, Y. Yue, and L. Qian, The Five-Hundred-Meter Aperture
Spherical Radio Telescope (FAST) Project, Int. J. Mod. Phys. D 20, 989 (2011).

[96] M. Favata, Conservative Self-Force Correction to the Innermost Stable Circular Orbit: Comparison with Multiple PostNewtonian-Based Methods, Phys. Rev. D 83, 024027 (2011). 